\title{
THE DISTRIBUTION OF DARK MATTER OVER THREE DECADES IN RADIUS IN THE LENSING CLUSTER
} ABELL 611

\author{
Andrew B. Newman ${ }^{1}$, Tommaso Treu ${ }^{2}$, Richard S. Ellis ${ }^{1}$, David J. SAND ${ }^{3,7}$, Johan Richard ${ }^{4}$, Philip J. Marshall ${ }^{2}$, \\ Peter CAPAK $^{5}$, ANd Satoshi Miyazaki ${ }^{6}$ \\ ${ }^{1}$ Department of Astronomy, California Institute of Technology, Pasadena, CA 91125, USA; anewman@ astro.caltech.edu \\ ${ }^{2}$ Department of Physics, University of California, Santa Barbara, CA 93106-9530, USA \\ ${ }^{3}$ Harvard-Smithsonian Center for Astrophysics, Cambridge, MA 02138, USA \\ ${ }^{4}$ Department of Physics, University of Durham, DH1 3LE, UK \\ ${ }^{5}$ Spitzer Science Center, Pasadena, CA 91125, USA \\ ${ }^{6}$ National Astronomical Observatory of Japan, Mitaka, Tokyo 181-8588, Japan \\ Received 2009 May 12; accepted 2009 September 21; published 2009 November 10
}

\begin{abstract}
We present a detailed analysis of the baryonic and dark matter distribution in the lensing cluster Abell 611 $(z=0.288)$, with the goal of determining the dark matter profile over an unprecedented range of cluster-centric distance. By combining three complementary probes of the mass distribution, weak lensing from multi-color Subaru imaging, strong lensing constraints based on the identification of multiply imaged sources in Hubble Space Telescope images, and resolved stellar velocity dispersion measures for the brightest cluster galaxy secured using the Keck telescope, we extend the methodology for separating the dark and baryonic mass components introduced by Sand et al. Our resulting dark matter profile samples the cluster from $\sim 3 \mathrm{kpc}$ to $3.25 \mathrm{Mpc}$, thereby providing an excellent basis for comparisons with recent numerical models. We demonstrate that only by combining our three observational techniques can degeneracies in constraining the form of the dark matter profile be broken on scales crucial for detailed comparisons with numerical simulations. Our analysis reveals that a simple Navarro-Frenk-White (NFW) profile is an unacceptable fit to our data. We confirm earlier claims based on less extensive analyses of other clusters that the inner profile of the dark matter profile deviates significantly from the NFW form and find a inner logarithmic slope $\beta$ flatter than $0.3\left(68 \%\right.$; where $\rho_{\mathrm{DM}} \propto r^{-\beta}$ at small radii). In order to reconcile our data with cluster formation in a $\Lambda$ CDM cosmology, we speculate that it may be necessary to revise our understanding of the nature of baryon-dark matter interactions in cluster cores. Comprehensive weak and strong lensing data, when coupled with kinematic information on the brightest cluster galaxy, can readily be applied to a larger sample of clusters to test the universality of these results.
\end{abstract}

Key words: dark matter - galaxies: clusters: individual (Abell 611) - galaxies: elliptical and lenticular, cD galaxies: formation - gravitational lensing

Online-only material: color figures

\section{INTRODUCTION}

The cold dark matter (CDM) model has been remarkably successful in explaining the observed large-scale structure of the universe (e.g., Springel et al. 2006). Cosmological $N$-body simulations have been a crucial tool in assessing CDM, making precise predictions of the growth of cosmic structure and the distribution of dark matter (DM) over a wide range of scales. The density profile of DM has particularly been the focus of intense theoretical and observational efforts, as it offers a tractable route toward testing the nature of dark matter and its interaction with baryons. Key issues include the form of the density profile, its universality over a wide range of halo masses, the degree of variance among halos, and the role of baryons in shaping the DM distribution.

Based on $N$-body simulations, Navarro et al. (1996, 1997, hereafter NFW) found that CDM halos are self-similar, differing only by simple rescalings of size and density, over four decades in mass. Within a scale radius $r_{s}$, this "universal" NFW density profile asymptotically approaches $\rho \propto r^{-\beta}$, with $\beta=1$; on larger scales, it approaches $\rho \propto r^{-3}$. Moreover, NFW found $r_{s}$ to be correlated with mass, implying that the diversity of

\footnotetext{
7 Harvard Center for Astrophysics and Las Cumbres Observatory Global Telescope Network Fellow.
}

DM halos is captured by a single parameter. Subsequent work with higher numerical resolution confirmed the basic findings of NFW (Moore et al. 1998; Ghigna et al. 2000; Diemand et al. 2004, 2005), but disagreed on the inner logarithmic slope $\beta$. Nonetheless, a steep ( $\beta \gtrsim 1)$ cusp was favored by all authors, with claims ranging up to $\beta \approx 1$.5. Subsequently, a generalized NFW (gNFW) profile was introduced:

$$
\rho(r)=\frac{\rho_{s}}{\left(r / r_{s}\right)^{\beta}\left(1+r / r_{s}\right)^{3-\beta}} .
$$

This reduces to the NFW form when $\beta=1$. More recent studies by Navarro et al. $(2004,2008)$ have hinted that, rather than converging to a power law, the density slope may become progressively shallower at very small radii $\lesssim 10^{-2} r_{s}$. These authors also emphasized slight differences in form among halos, demonstrating that self-similarity may not strictly be the case; however, it must be emphasized that these deviations from NFW, although significantly determined, are still small.

Despite its triumphs on cosmological scales, CDM predictions on galaxy scales have often been difficult to reconcile with observations. A particular challenge is the high central density expected in DM halos. Studies of dwarf and low surface brightness galaxies, which are expected to be DM dominated throughout, reveal kinematics incompatible with CDM halos 
(e.g., Côté et al. 2000; Marchesini et al. 2002; Simon et al. 2003; Gentile et al. 2005; Kuzio de Naray et al. 2008, but see Swaters et al. 2003). These data are often more consistent with a shallow cusp $(\beta<1)$ or cored $(\beta=0)$ density profile than with an NFW-like cusp. Rotation curves of spiral galaxies have also indicated lower central densities than are expected in CDM halos (e.g., Navarro \& Steinmetz 2000; Mo \& Mao 2000; Salucci 2001; Binney \& Evans 2001; Alam et al. 2002; McGaugh et al. 2007). It is generally thought that accounting for baryons will make simulated halos even more concentrated; we discuss the current literature in Section 6, including contrary indications.

Thorough observational testing of the numerical simulations requires checking their predictions on all scales. Galaxy clusters provide an excellent laboratory for these tests because several independent observational probes are available, spanning a large dynamic range in density and cluster-centric radius. The mass profile of clusters has been a hotly debated topic in recent years. The inner slope $\beta$ and the concentration parameter $c=r_{\text {vir }} / r_{s}$ have been particularly controversial, with different groups obtaining results ranging from perfect agreement to highly significant disagreement between observations and CDM predictions, often for the very same clusters (e.g., Smith et al. 2005; Gavazzi 2005; Zappacosta et al. 2006; Schmidt \& Allen 2007; Umetsu \& Broadhurst 2008; Bradač et al. 2008; Limousin et al. 2008).

In addition to scatter in the mass profile from cluster to cluster and possible selection biases, other factors clearly contribute to this broad and often conflicting range of results. First, different groups adopt different definitions for the inner slope $\beta$; some use it to refer to the total mass density profile, whereas others use it to refer only to that of the dark matter as we do in this paper. Second, the stellar mass of the central galaxy is often neglected on the ground that it makes a negligible contribution to the total mass. However, this is not the case in the innermost regions where stars typically dominate the density profile. Third, studies that rely on an individual observational technique cover a limited dynamic range in radius, thus effectively relying on an NFW (or other) model to extrapolate the results well beyond the region probed by the data.

$\mathrm{X}$-ray temperature measurements typically reach out to $\sim 500 \mathrm{kpc}$ (e.g., Bradač et al. 2008), and are often limited to radii larger than $\sim 50 \mathrm{kpc}$ due to instrumental resolution or residual substructure (e.g., Schmidt \& Allen 2007). Strong lensing is typically sensitive to the projected mass distribution inside $\sim 100-200 \mathrm{kpc}$, with limits on at $\sim 10-20 \mathrm{kpc}$ available in only the most favorable cases (e.g., Gavazzi 2005; Limousin et al. 2008). Weak lensing requires averaging noisy signal from many background galaxies and therefore does not have the resolution to constrain profiles inside $\sim 100 \mathrm{kpc}$. Stellar kinematics of the central galaxy can cover the $\sim 1-200 \mathrm{kpc}$ region with long exposures on large telescopes (Kelson et al. 2002). Satellite kinematics is typically limited to radii larger than $\sim 100-200 \mathrm{kpc}$ (e.g., Diaferio et al. 2005; Biviano \& Salucci 2006). It is thus clear that only by combining multiple diagnostics (e.g., Miralda-Escude 1995; Sand et al. 2002; Kneib et al. 2003; Bradač et al. 2005; Mahdavi et al. 2007) can one hope to achieve a precise and accurate determination of the inner slope, and thus assess the validity of the NFW profile.

With this goal in mind, Sand et al. (2002, 2004) studied a sample of nearly round, apparently "relaxed" clusters by combining strong gravitational lensing constraints with the velocity dispersion profile of the central brightest cluster galaxy (BCG). The combination of the two techniques allowed them to dis- entangle the contribution of the stellar mass of the BCG from that of the dark matter halo and to obtain constraints over almost two decades in radius. They found that the steep cusps produced in $N$-body simulations were inconsistent with the data, and instead favored a shallower slope with $\langle\beta\rangle=0.52 \pm 0.05 \pm 0.2$ (statistical and systematic errors). Following claims by some workers that this discrepancy could be an artefact of simplifying assumptions in the analysis, such as negligible ellipticity (Bartelmann \& Meneghetti 2004; Meneghetti et al. 2007), Sand et al. (2008) presented a more sophisticated, fully twodimensional analysis of two clusters. They reached similar conclusions (e.g., $\beta=0.45_{-0.25}^{+0.2}$ for Abell 383), but again noted degeneracies (e.g., between $r_{s}$ and $\beta$ ) that could only be broken by including mass tracers at larger radii. This paper is the next step in a continuing effort to build on the work of Sand et al. (2002, 2004, 2008). We are currently collecting data for a sample of 10 clusters, and present here a pilot study of Abell $611(z=0.288$, Crawford et al. 1995). This cluster exhibits a regular, nearly round morphology in X-ray and optical imaging. In addition to the strong lensing constraints (30-90 kpc) and stellar kinematics $(\lesssim 20 \mathrm{kpc})$, we have obtained wide-field Subaru imaging to measure mass on $0.15-3 \mathrm{Mpc}$ scales via weak lensing, thus breaking the remaining degeneracies noted in Sand et al. (2008). The goals of this paper are to determine whether an NFW profile provides an acceptable fit to all constraints, whether the gNFW profile substantially improves the fit, and if so, the range of inner slopes $\beta$ that is permitted.

A full comparison of observational data with numerical simulations is possible only by probing mass on all scales. It is much easier for theory to fit data acceptably in a single regime (e.g., on weak or strong lensing scales alone) than over the full extent of the cluster. We thus consider the wide dynamic range of our data (three decades in radius) to be a prime advantage of our analysis. Additionally, a proper comparison with theory requires separating DM from baryons. Although baryons are a small fraction of the total cluster mass, they can dominate on kpc scales. We therefore carefully model starlight in the $\mathrm{BCG}$ to account properly for stellar mass.

The paper is organized as follows. The next three sections (Sections 2 to 4 ) describe the three main data sets used in the analysis. Each data set is the foundation of one of three mass probes that are combined in this paper. Discussing each one separately allows us to present the measurements in detail and the strengths and weaknesses of the probe: Section 2 presents a weak-lensing analysis based on wide-field Subaru imaging; Section 3 presents a strong lensing analysis based on Hubble Space Telescope data, which is also used to constrain the distribution of starlight on galactic scales; Section 4 presents a dynamical analysis of the stellar kinematics in the BCG obtained from Keck spectroscopy. Our results on the dark matter density profile of the cluster based on the combination of the three probes are presented in Section 5, illustrating how this powerful combination breaks the degeneracies inherent to each method alone. Limitations of our analysis and residual systematic effects are also considered. Section 6 discusses our results in the context of the literature and Section 7 summarizes.

Throughout the paper, we adopt the cosmological parameters $\left(h, \Omega_{m}, \Omega_{v}\right)=(0.7,0.3,0.7)$. Magnitudes are given in the AB system unless otherwise stated. Marginalized posterior distributions are characterized by the mode, and error bars refer to the $68 \%$ confidence level. 


\section{SUBARU IMAGING AND WEAK LENSING ANALYSIS}

This section presents an analysis of wide-field, multi-color Subaru imaging data whose purpose is to measure, via the gravitational shear, the radial mass distribution from $150 \mathrm{kpc}$ to $3.25 \mathrm{Mpc}$. The shear signal is limited outside this interval by the surface density of suitable background galaxies on small scales, and by confusion with large-scale structure on large scales. We use photometric redshifts to identify foreground and cluster member galaxies, which are not lensed by the cluster and so dilute the shear signal if not carefully excluded. Shapes of galaxies are measured and corrected for atmospheric and instrumental distortions using the method originally outlined by Kaiser et al. (1995, hereafter KSB). We have calibrated this procedure using simulated data. The two-dimensional mass map reveals no significant secondary mass concentrations, justifying our use of simple parametric models. We show that our radial mass profile is in excellent agreement with independent X-ray observations.

\subsection{Observations and Data Reduction}

We observed Abell 611 on 2007 November 12-13 using SuprimeCam at the Subaru Telescope under excellent conditions. $B_{J} V_{J} R_{C} I_{C}$ imaging was obtained, with $1200 \mathrm{~s}$ integration in $V_{J}$ and $2400 \mathrm{~s}$ in each of $B_{J} R_{C} I_{C}$. We conduct shape measurement in the $R_{C}$ imaging; the remaining filters are used for photometric redshifts. The median stellar FWHM in the $R_{C}$ imaging is $0^{\prime \prime} .7$, and the $1 \sigma$ surface brightness limit is 27.2 mag $\operatorname{arcsec}^{-2}$.

The SuprimeCam imaging was reduced using the IMCAT $^{8}$ software. One corner CCD (DET-ID 0) was excluded from our analysis due to poor charge transfer efficiency. Bias was measured from the median of six frames and subtracted. Saturated pixels, chip defects, ghosts, and satellite trails were masked, and cosmic rays were rejected using gethotpix. Since scattered light is significant and non-uniform across the camera, flat-fielding must be conducted on a chip-by-chip basis. In each filter, a flat was constructed from the night sky imaging by placing large elliptical masks around objects detected by hfindpeaks, normalizing each frame by its median, and then median-combining all frames with a $2 \sigma$ clip. In order to have enough frames for this procedure to be effective, we combined data from both nights. The chip-to-chip normalization was provided by the SuprimeCam team (H. Furusawa \& Y. Komiyama 2008, private communication).

An initial sky subtraction is made by subtracting the mode of each chip. However, large-scale gradients remain from residual scattered light and Galactic cirrus. We therefore adopt a more sophisticated background subtraction scheme adapted from that described by Donovan (2007) and Capak et al. (2007). Roughly, each chip is divided into a grid, and the background is measured in each cell after excluding objects. The cells are interpolated via Delaunay tesselation to form a continuous background image, which is then subtracted. This method is successful at removing background gradients, stellar halos, and charge buildup.

Photometry was calibrated using two Landolt fields. Hervé Aussel kindly provided AB magnitudes in the SuprimeCam filters for our fields, using fits to PHOENIX stellar atmosphere models (Hauschildt et al. 1997) with UBVRI (Landolt 1992), ugriz (Adelman-McCarthy et al. 2008, SDSS DR6), and JHK (Skrutskie et al. 2006, 2MASS) photometry. We used five stars

\footnotetext{
8 http://www.ifa.hawaii.edu/ kaiser/imcat/
}

with well-fit spectral energy distributions (SEDs) to measure the photometric zero point, and typical Mauna Kea values for the air mass correction, which was always $\lesssim 0.03 \mathrm{mag}$.

The astrometric solution was obtained by fitting the positions of unsaturated stars to a low-order polynomial with iterative rejection. The median dispersion among stellar positions in different exposures was $0{ }^{\prime \prime} .007$. Absolute astrometry (tangent point, rotation, scale) was anchored to stars in the USNO-B catalog (Monet et al. 2003). Images were placed on a stereographic projection and combined using a clipped weighted mean.

Noise maps were created by measuring the variance in each chip (before applying distortion) and propagating through the co-addition. Diffraction spikes, halos and rings of very bright stars $(V \lesssim 10)$, and perimeter regions were masked in the stacked image.

\subsection{Source Catalog and Photometric Redshifts}

Object detection was performed using SExTRACTOR (Bertin $\&$ Arnouts 1996) with a threshold of $0.5 \sigma$ over $\geqslant 15$ contiguous pixels. Extinction was corrected in each filter using the reddening map of Schlegel et al. (1998) and the Galactic dust law. Colors were measured in $3^{\prime \prime}$ apertures, with aperture corrections determined using bright stars.

The BPZ (Benítez 2000) code was used to obtain photometric redshifts using the $R_{C}$-band MAG_AUTO magnitude and the color measurements described above. The CWWSB templates and a prior on the luminosity function from the Hubble Deep Field (HDF) were used. For our weak lensing galaxy sample, cuts are placed on $z_{b}$, the marginalized redshift, and on $P(z>$ 0.4 ), the probability that $z>0.4$. We require $0.4<z_{b}<1.3$, with the upper limit being roughly the redshift at which the $4000 \AA$ feature redshifts outside the $I_{C}$ filter and beyond which there is significant degeneracy with low-redshift galaxies. We also require $P(z>0.4)>0.75$; this cut was determined from Monte Carlo simulations by requiring $90 \%$ confidence in $<10 \%$ contamination by unlensed galaxies in the inner cluster ( $<250 \mathrm{kpc}$ projected radius).

We have checked the redshift distribution we obtain in Abell 611 against those obtained in other Subaru fields with wider wavelength coverage, as shown in Figure 1. The redshift distribution for Abell 611 is bracketed by those of the four comparison fields, with no obvious systematic errors within the limits imposed by cosmic variance. For each field, we have also calculated the effective redshift $z_{e}$, at which the lensing efficiency $D_{l s} / D_{s}$ equals the sample mean, restricting to $0.5 \leqslant z \leqslant 1.3$. (The lower limit is to avoid the cluster in Abell 370; the upper limit is that which we adopted in Abell 611.) For Abell $611 z_{e}=0.79$, in good agreement with the other fields: $z_{e}=0.78(\mathrm{~A} 370), z_{e}=0.82(\mathrm{HDF}), z_{e}=0.82(\mathrm{LH}-\mathrm{NW})$, $z_{e}=0.89$ (SSA22). We see no evidence that our $B V R I$ coverage is insufficient to provide redshifts of sufficient accuracy in the restricted range $z<1.3$. Since our data and the reference fields have been analyzed similarly, in particular using the same BPZ code, they are potentially subject to common systematic errors. Other studies have found that BPZ performs well in comparison to large spectroscopic samples (e.g., Mobasher et al. 2007).

We conclude that $z_{e}$ is unlikely to be in error by more than 0.03 , corresponding to an error of $2 \%$ in the shear normalization. The propagation of this uncertainty is discussed in Sections 2.4 and 2.5 . 


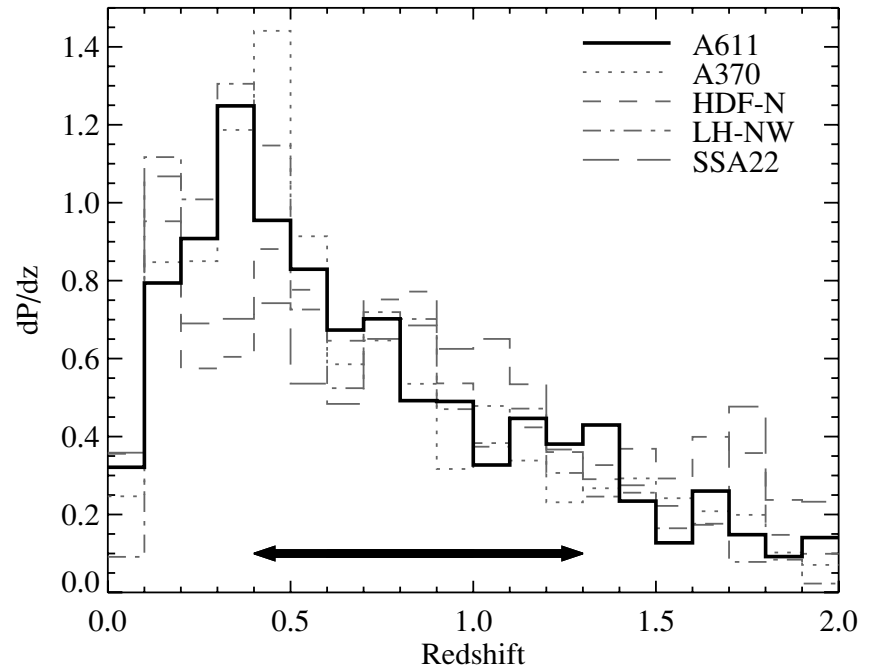

Figure 1. Redshift probability density for $R<25.8(\simeq 5 \sigma)$ galaxies in Abell 611, as well as 4 comparison fields also obtained with Subaru, but with wider wavelength coverage: A370 (UBVRIZ), HDF-N (UBVRIZ, HK, NB816), Lockman Hole Northwest ( $B V R I Z, H K)$, SSA22 (UBVRIZJHK). Note spikes in the A611 $(z=0.3)$ and A370 $(z=0.4)$ fields at the cluster redshifts. Our redshift distribution is generally bracketed by the comparison fields and appears consistent within the limits of cosmic variance. Galaxies used in our weak lensing analysis lie in the redshift interval indicated by the arrow; however, the additional cut $P(z>0.4)>0.75$ eliminates most galaxies with $z \lesssim 0.5$.

\subsection{Galaxy Selection and Shear Measurement}

Shapes of galaxies must be corrected for both distortions introduced by the point-spread function (PSF), which is generally anisotropic and varies across the focal plane, as well as the isotropic smearing introduced both by the atmosphere and by the smoothing kernel used in our shape measurements. The determination of the PSF from stellar images and its application to correcting galaxy shapes is described in Appendix A. The interested reader can also find details of our measurement of the reduced shear $g_{\alpha}$ in Appendix B. Here, we describe only the weak lensing sample selection.

Proper measurement of the weak gravitational shear requires careful selection of the lensed galaxy sample, avoiding contamination by cluster members and foreground objects which could dilute the shear signal. We adopt the following cuts to remove spurious detections and select galaxies that are resolved, significantly detected, uncontaminated by neighbors, and located well behind the cluster:

1. $1.1\left\langle r_{h}^{*}\right\rangle<r_{h}<6$ pixels, where $r_{h}$ is the half-light radius and $\left\langle r_{h}^{*}\right\rangle$ is the median stellar half-light radius. The lower limit selects resolved objects; the upper limit removes foreground objects and spurious detections.

2. Positive flux detected with $\mathrm{S} / \mathrm{N}=$ FLUX_AUTO/ FLUXERR_AUTO $>5$, which tests on STEP simulations (Section 2.4) showed to be a reasonable threshold.

3. $R_{C}>21 \mathrm{mag}$, approximately the saturation limit.

4. PSF-corrected ellipticity $\left|e^{\prime}\right|<1$, since in the weak-lensing regime, higher ellipticities often indicate blends.

5. No masked pixels, or pixels assigned by SExTRACTOR to nearby neighbors, within $2 r_{g}$ (defined in the Appendix).

6. $\operatorname{tr} P_{\mathrm{sh}}>0$ and $\operatorname{tr} P_{\mathrm{Sm}}>0$, as defined in the Appendix.

7. $d<0.2$ pixels, where $d$ is the distance between the centroids measured with and without smoothing. For symmetric objects, $d=0$ in the absence of noise. We find that large separations usually indicate blended or asymmetric objects.

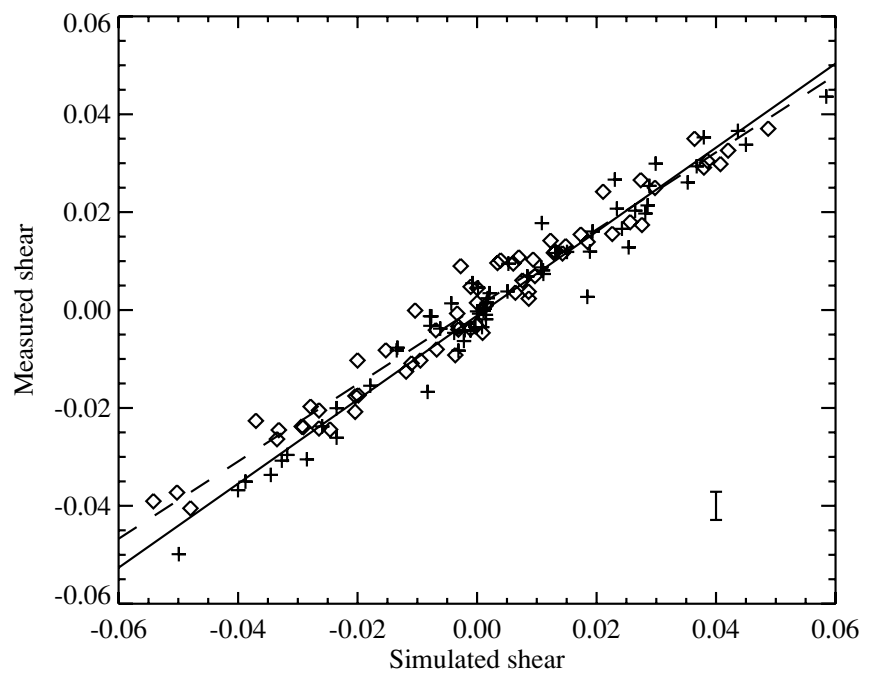

Figure 2. Response of our shear measurement technique to simulated shear fields from STEP2 PSF A (0.'6). Crosses and diamonds denote the $g_{1}$ and $g_{2}$ components, which are best fit by the solid and dashed lines, respectively. A typical error bar is shown.

8. $0.4<z<1.3$ and $P(z>0.4)>0.75$, as discussed in Section 2.2.

This selection yields a galaxy density of $14.5 \operatorname{arcmin}^{-2}$, comparable to other studies with the Subaru telescope (e.g., Umetsu et al. 2009).

\subsection{Shear Calibration}

The KSB method is known to underestimate shear by $\sim 10 \%$ $15 \%$ (e.g., Erben et al. 2001). We therefore calibrated our method using the STEP2 simulation (Massey et al. 2007), in which shapelet models of galaxies are subjected to an anisotropic PSF and known, constant shear and placed in an image designed to mimic Subaru data. PSF models A and C have sizes $\sim 0$.' 6 and $\sim 0^{\prime \prime}$, 8 , respectively, which bracket the $0^{\prime \prime}$.7 seeing in our data. We measured the shear in 128 simulated fields (64 per PSF model) using the same method applied to the real data, with a few exceptions. First, noise was estimated by SExTRACTOR from the variance in the co-added image and corrected upward to account for noise correlation. Also, orders of the interpolating polynomials were reduced owning to the smaller $7^{\prime}$ field containing fewer galaxies.

For each field, the weighted mean shear and its uncertainty were estimated and are shown as a function of the true shear for PSF A in Figure 2. (STEP2 simulations have zero convergence, so there is no distinction between shear and reduced shear.) A linear relation

$$
g_{\alpha, \text { meas }}=m g_{\alpha, \text { input }}+c
$$

was fitted to both shear components of each PSF model. We note no signs of non-linear response in this shear regime. The fit parameters $m$ and $c$ are given in Table 1. Additive error reflected in $c$ results from error in the PSF determination and is $\lesssim 10^{-3}$; we conclude that our correction for PSF anisotropy is accurate. Multiplicative calibration error is reflected in $m$. The mean and standard deviations of these measurements is $m=0.81 \pm 0.03$, and we correct all shear measurements and associated uncertainties in this work by this factor. This is within the range of $m$ reported for STEP2 participants (Massey et al. 2007), although rather at the low end. 
Table 1

Shear Calibration With STEP2

\begin{tabular}{lcrc}
\hline \hline PSF & Component & \multicolumn{1}{c}{$c$} & \multicolumn{1}{c}{$m$} \\
\hline A & $g_{1}$ & $(-1.1 \pm 0.7) \times 10^{-3}$ & $0.86 \pm 0.03$ \\
A & $g_{2}$ & $(0.7 \pm 0.7) \times 10^{-3}$ & $0.79 \pm 0.03$ \\
C & $g_{1}$ & $(2.3 \pm 0.7) \times 10^{-3}$ & $0.80 \pm 0.03$ \\
C & $g_{2}$ & $(0.5 \pm 0.7) \times 10^{-3}$ & $0.80 \pm 0.03$ \\
\hline Average & & & $0.81 \pm 0.03$
\end{tabular}

Note. Errors are obtained from least-squares linear fitting. The rms residual in each case is 0.004

\subsection{The Mass Distribution from $150 \mathrm{kpc}$ to $3.25 \mathrm{Mpc}$}

We use the LENSENT2 code (Marshall et al. 2002) to produce a mass map and verify that the dark matter distribution in Abell 611 is apparently relaxed, symmetric, and centered on the BCG. A Gaussian intrinsic correlation function (ICF) with a FWHM of $170^{\prime \prime}$ was used, since this maximises the detection significance; we also note that larger ICFs do not significantly increase the evidence. Figure 3 shows the smoothed light distribution and mass contours. The offset between the light and mass centers is within the bin size used in the mass reconstruction, and we thus consider it insignificant. In addition to the cluster core, a few other mass concentrations are marginally detected. The most significant of these is approximately $1.5 \mathrm{Mpc}$ north. The excess mass in this clump is $\lesssim 10^{14} M_{\odot}$, or $\lesssim 15 \%$ of the cluster virial mass, and the detection is $<3 \sigma$. Our treatment of this clump is discussed further below.

Having established that the weak lensing signal can be described by a single concentration of mass, we now proceed to an analysis based on simply parametrized models. For this purpose, we consider only the signal arising from background galaxies whose projected distance from the cluster center is between $150 \mathrm{kpc}$ and $3.25 \mathrm{Mpc}$. The outer limit is motivated by the onset of contamination from large-scale structure (Hoekstra 2003). We adopt the inner limit to avoid the strong-shear regime, unprobed by STEP2, where non-linear calibration errors may become significant; in any case, the number of selected galaxies within $150 \mathrm{kpc}$ is very small. The individual photometric redshifts of sources are used in our shear calculations.

Throughout this paper, we use a modified version of the LENSTOOL code ${ }^{9}$ (Jullo et al. 2007; Kneib et al. 1993) to fit data to parametrized models. Samples are drawn from 10 parallel chains using the Markov Chain Monte Carlo (MCMC) technique with simulated annealing. The rate parameter, which controls the convergence speed, was set to 0.05 in order to encourage full exploration of the parameter space, and to obtain sufficiently precise measures of the evidence with reasonable computational effort. In all cases, we repeat the MCMC analysis 4 times to estimate the error on the Bayesian evidence. Parameters were estimated by combining chains, using at least 6000 samples.

For comparison with the literature and with theory, we fit the shear data to a pseudoelliptical NFW profile. Since the mass of cluster galaxies is negligible on the large scales at which we measure shear, the mass model includes only the cluster-scale DM halo. The shear calibration $m$ is included as a free parameter, with a prior based on the STEP2 results of Section 2.4 , thereby propagating the calibration uncertainty. Recognizing the potential for small systematic redshift errors that mimic shear calibration error (Section 2.2), as well as the

\footnotetext{
9 http://www.oamp.fr/cosmology/lenstool/
}

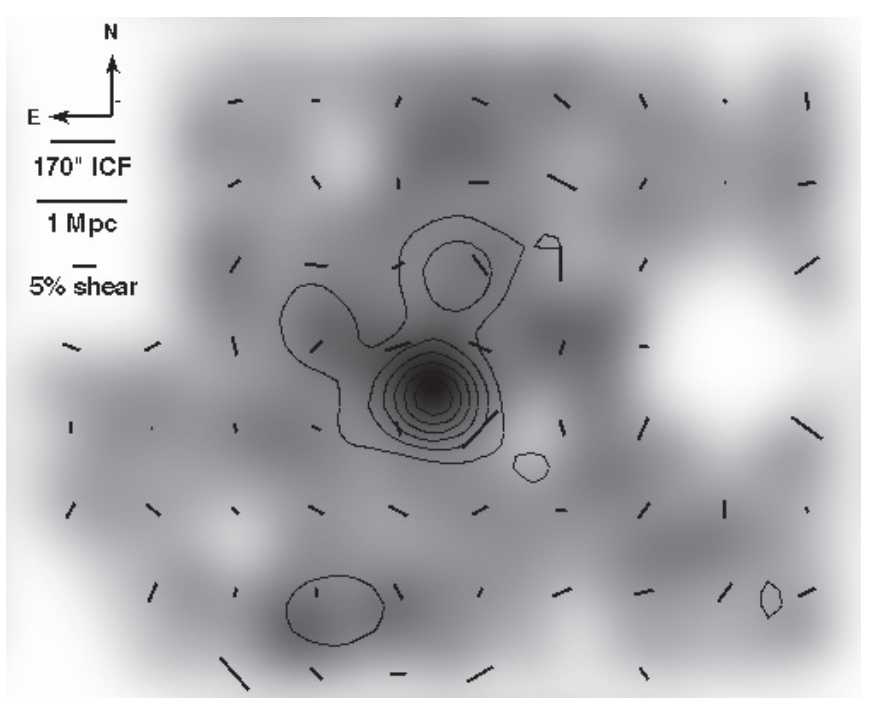

Figure 3. Smoothed galaxy light distribution with shear field and weak lensing mass contours overlaid. Contours are linear in mass, with the lowest corresponding to $\sim 2 \sigma$. Light regions represent those around masked bright stars. Within the uncertainty from binning, the mass and light centroids are coincident. The width of the ICF used in the mass reconstruction is shown; the light is smoothed to the same scale.

systematic uncertainties inherent in applying simulation-based calibrations to real data, we have slightly inflated the error in $m$ to 0.05 . For each model suggested by the MCMC sampler, the reduced shear polar $\mathbf{g}_{i, \text { model }}$ is computed at the position of each galaxy $i$, and

$$
\chi_{\mathrm{WL}}^{2}=\sum_{i}\left|\frac{\mathbf{g}_{i, \text { meas }} / m-\mathbf{g}_{i, \text { model }}}{\sigma_{i} / m}\right|^{2}
$$

is calculated, where $\sigma_{i}$ is the shear uncertainty described in Appendix B.

The LENSTOOL code parameterizes the NFW profile with the canonical scale radius $r_{s}$ and $\sigma_{0, \mathrm{DM}}$, a characteristic velocity dispersion defined by

$$
\sigma_{0, \mathrm{DM}}^{2}=\frac{8}{3} G \rho_{s} r_{s}^{2} .
$$

The results of the fit are presented in Table 2 and expressed both in terms of LENSTOOL parameters and in the standard parameterization of the NFW profile using $M_{200}$ and $c_{200} .{ }^{10}$ The measured radial shear profile is compared with the results of the fit in Figure 4. The $B$-mode signal-consistent with zero-is also shown, indicating that systematic errors are smaller than statistical errors.

We have experimented with a two-component mass model incorporating the marginally-detected clump previously noted in our two-dimensional mass reconstruction. However, $\chi_{\mathrm{WL}}^{2}$ is not improved, and the Bayesian evidence is much reduced, strongly arguing against the need for modeling the clump. We therefore do not consider any additional large-scale mass components for the remainder of this paper. We also note that assuming axial symmetry, rather than the two-dimensional elliptical symmetry we adopt here for consistency, has a negligible effect on the inferred parameters.

\footnotetext{
10 When elliptical models are considered, we use a circularized measure for
} the radii $r_{200}$ and $r_{s}$, and hence $c_{200}=r_{200} / r_{s}$. 


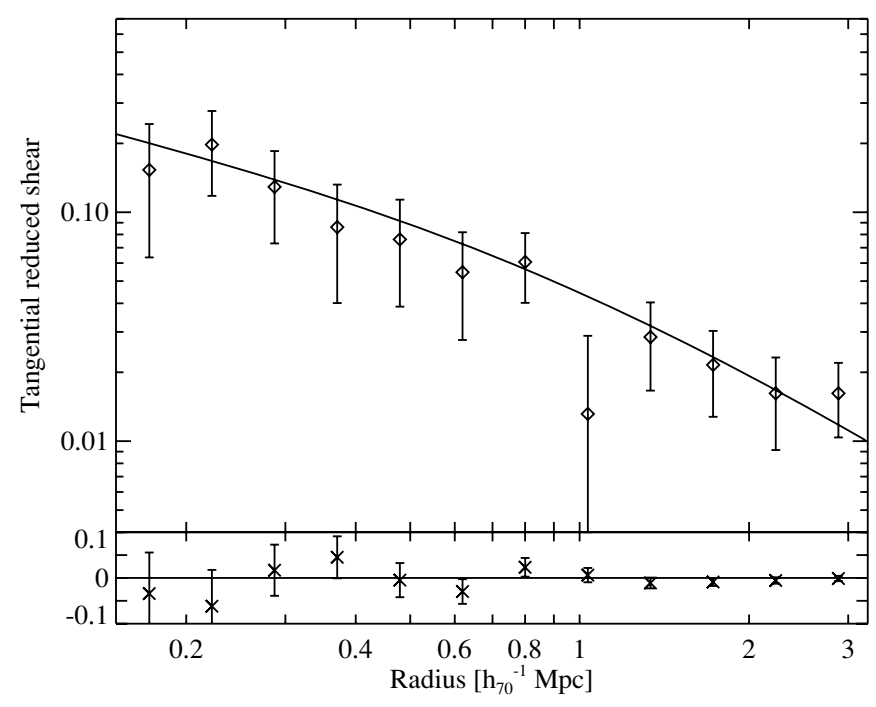

Figure 4. Top: radial profile of tangential reduced shear and the best-fitting NFW model (Table 2). Bottom: $B$-mode signal, consistent with zero $\left(\chi^{2} /\right.$ dof $=$ $6646 / 6545=1.02)$.

Table 2

NFW Fit to Weak Lensing Data

\begin{tabular}{lccc}
\hline \hline Parameter & Units & Prior & Posterior \\
\hline$\epsilon$ & $\ldots$ & {$[0,0.3]$} & $<0.16$ \\
P.A. & $\mathrm{deg}$ & {$[0,180]$} & $28 \pm 26$ \\
$r_{s}$ & $\mathrm{kpc}$ & {$[50,800]$} & $301_{-102}^{+189}$ \\
$\sigma_{0, \mathrm{DM}}$ & $\mathrm{km} \mathrm{s}^{-1}$ & {$[1000,2200]$} & $1525 \pm 110$ \\
$M_{200}$ & $10^{14} M_{\odot}$ & $\ldots$ & $8.0_{-1.8}^{+2.9}$ \\
$c_{200}$ & $\ldots$ & $\ldots$ & $4.7_{-1.2}^{+2.7}$ \\
$m$ & $\ldots$ & $(\mu, \sigma)=(0.81,0.05)$ & $0.80 \pm 0.06$ \\
\hline \multicolumn{2}{l}{ Minimum $\chi_{\mathrm{WL}}^{2}(13090$ constraints $)$} & & 13083.2 \\
\hline
\end{tabular}

Notes. Brackets specify the range of uniform priors. For the shear calibration $m$ (Equation (2)), a Gaussian prior was used. $\sigma_{0, \text { DM }}$ is defined in Equation (4). $\epsilon$ is the pseudoellipticity of Golse \& Kneib (2002).

Our results are in excellent agreement with $\mathrm{X}$-ray measurements by Schmidt \& Allen (2007), who found $r_{s}=320_{-110}^{+240} \mathrm{kpc}$ and $c_{200}=5.1_{-1.6}^{+1.7}$; this again suggests there are no significant systematic errors in our weak lensing analysis. The concentration $c_{200}=4.7_{-1.2}^{+2.7}$ is consistent with the CDM expectation of $4.1 \pm 0.4$ (Neto et al. 2007) for a "relaxed" halo of this mass.

\section{HST IMAGING, SURFACE PHOTOMETRY, AND STRONG LENSING ANALYSIS}

\subsection{Observations and Data Reduction}

Abell 611 was observed by HST/ACS with a 36 minute integration in the F606W filter (ID 9270, PI: Allen). We obtained the data from the HST archive in reduced and drizzled form; however, the background level was not exactly uniform among chips. We thus remeasured and subtracted the background in the two relevant chips, taking care to avoid bias from the extended halos of galaxies, particularly the BCG.

\subsection{BCG Surface Brightness Profile}

The distribution of stellar mass in the BCG must be measured to model strongly lensed features and stellar velocity dispersions. The BCG surface brightness profile from HST imaging was fit to an elliptical $R^{1 / 4}$ profile using the two-dimensional GALFIT code (Peng et al. 2002). Cluster galaxies were excluded
Table 3

BCG Surface Brightness Profile

\begin{tabular}{lccccc}
\hline \hline Model & $R_{e}$ or $r_{\text {cut }}$ & $r_{\text {core }}$ & $m_{\mathrm{F} 606 \mathrm{~W}}$ & $b / a$ & P.A. \\
\hline$R^{1 / 4}$ & $9.79 \pm 1.3$ & $\ldots$ & $16.77 \pm 0.12$ & $0.720 \pm 0.008$ & $42.7 \pm 0.6$ \\
dPIE & $10.0 \pm 0.8$ & $0.25 \pm 0.01$ & $16.88 \pm 0.14$ & $\cdots$ & $\cdots$ \\
\hline
\end{tabular}

Notes. Distances are in arcseconds. The scale is $4.33 \mathrm{kpc} \operatorname{arcsec}^{-1}$. The $R_{e}$ parameter from GALFIT has been circularized by multiplying by $(b / a)^{1 / 2}$. Uncertainties are primarily systematic and were estimated by varying the sky level and fitting region.

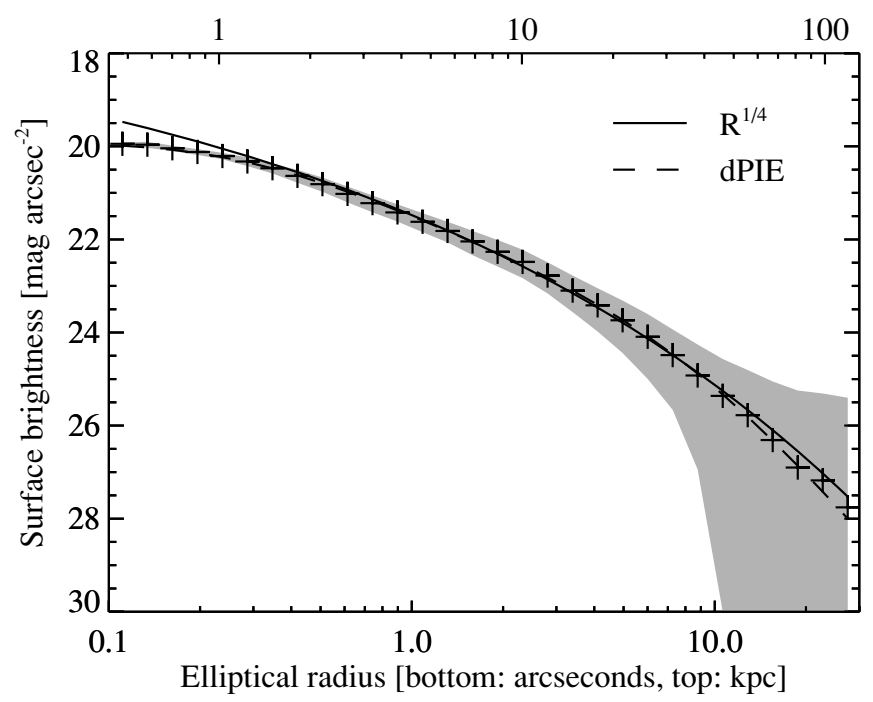

Figure 5. Radial surface brightness profile of the Abell 611 BCG with PSFconvolved $R^{1 / 4}$ and dPIE fits. The mean surface brightness in radial bins is denoted with crosses, and the $1 \sigma$ dispersion in each data bin is shaded. The $R^{1 / 4}$ and dPIE fits are used in modeling the strong lensing and velocity dispersion constraints, respectively. Here the elliptical radius is defined by $r^{2}=x^{2} /(1+e)^{2}+y^{2} /(1-e)^{2}$, where $e=\left(a^{2}-b^{2}\right) /\left(a^{2}+b^{2}\right)$.

from the fit by enlarging their SExTRACTOR ellipses by a factor of 5; extended arcs and diffraction spikes were masked manually. The PSF was accounted for using a TINYTIM model (Krist 1993), and variance was estimated from standard CCD parameters scaled by the exposure map. The fit parameters are given in Table 3. The uncertainties are dominated by systematic effects, primarily the sky level and the fitting region. Both were varied to obtain the estimates in Table 3.

We use the $R^{1 / 4}$ fit in our dynamics modeling, and Figure 5 shows that this indeed provides a good description of the data in the $\sim 3-20 \mathrm{kpc}$ region over which we measure velocity dispersions. For strong lensing purposes, we adopt the dual pseudoisothermal elliptical mass distribution (dPIE),${ }^{11}$ which is characterized by two length scales $r_{\text {core }}$ and $r_{\text {cut }}$, with $r_{\text {core }}<$ $r_{\text {cut }}$. The dPIE model in Table 3 was obtained by adopting the ellipticity parameters from GALFIT and fitting the surface brightness as a function of elliptical radius. As shown in Figure 5, this is a excellent fit over the entire strong lensing regime. We determine the total luminosity from the dPIE model. Galactic extinction was corrected using the $E(B-V)$ values and extinction coefficients of Schlegel et al. (1998). Tranforming to the Vega system, and using the transformation $\Delta m_{\mathrm{B}-\mathrm{F} 606 \mathrm{~W}}=0.65 \pm 0.05$ calculated by Treu et al. (1999) for a $z=0.292$ passive galaxy, we find $M_{\mathrm{B}, \text { Vega }}=-23.89 \pm 0.15$ and $L_{\mathrm{B}}=(5.6 \pm 0.8) \times 10^{11} L_{\odot}$.

\footnotetext{
11 Also called truncated PIEMD (see Elíasdóttir et al. 2007) and distinct from
} the original model of Kassiola \& Kovner (1993). 


\subsection{Multiple Image Constraints}

The mass distribution from $\sim 30-90 \mathrm{kpc}$ is constrained by three multiply-imaged sources identified in Figure 6. Spectroscopic redshifts are available for sources 1 and 2 (Richard et al. 2009). We conservatively adopted as constraints only the multiple images we consider most secure. However, objects are detected at all locations of counter images predicted by the model, providing a vital check.

1. Source 1. Five images are identified by a complex morphology with five distinguishable knots per image. We chose to include only one knot (knot A of Richard et al. 2009) as a constraint, both to avoid overweighting these images and for computational efficiency. We have verified that including all knots does not significantly affect our results. Images 1.4 and 1.5 are split by the galaxy they bracket, with a likely radial counter image indicated at the bottom of the inset in Figure 6. Although this image is predicted by our model, the predicted position is too uncertain to make a secure identification, and we therefore decline to include this image as a constraint.

2. Source 2. This source forms a giant tangential arc. Three merging images are identified from brightness peaks.

3. Source 3. Images 3.1 and 3.2 are identified by their common and distinct "horseshoe" morphology. The redshift has not been measured spectroscopically, and the faintness of the images, along with contamination from other sources, precludes accurate color measurements in our ground-based data. Three additional images are predicted by our initial models; two of these (3.3 and 3.4) have predicted positions and fluxes that match well with features in the HST imaging, and we do include these as constraints. The third predicted image is in the cluster core, and although we identify a potential match in the inset of Figure 6, we do not consider the interpretation reliable enough for use as a constraint.

In total, we use $n_{i}=12$ images of $n_{s}=3$ sources, resulting in $2\left(n_{i}-n_{s}\right)=18$ constraints on our model.

\subsection{Cluster Member Galaxies}

Cluster galaxies other than the BCG can perturb the positions of nearby images. These are included in our strong lens model via dPIE profiles, with properties set by scaling relations that are motivated by the fundamental plane. The cluster member catalog was selected from resolved HST sources, detected using SExTRACTOR, that have (1) projected clustercentric radius $<400 \mathrm{kpc}$ (imaging is complete to $\sim 200 \mathrm{kpc}$ ), (2) $\left|z_{\text {phot }}-z_{\text {cluster }}\right|<0.2(\sim 2 \sigma)$, (3) and $m_{\mathrm{F} 606 \mathrm{~W}}<23$. This selects 90 galaxies; an additional fainter perturber near image 1.3 was also included. SEXTRACTOR centroids and ellipticities were used, while the dPIE parameters $r_{\text {cut }}$ and $\sigma_{0}$ were set by scaling relations. We adopted the $r^{*}$-band relation $L / L_{*}=\left(\sigma_{0} / \sigma_{0 *}\right)^{3.91}$ found by Bernardi et al. (2003) for early-type SDSS galaxies, where $M_{*}=-21.39$ and $\sigma_{0 *}=158 \mathrm{~km} \mathrm{~s}^{-1}$ at the cluster redshift, accounting for luminosity evolution. However, recognizing the $\sim 25 \%$ intrinsic scatter in this relation and possible systematic errors (e.g., photometry errors within the BCG), $\sigma_{0 *}$ was allowed to vary by imposing a Gaussian prior with a $50 \mathrm{~km} \mathrm{~s}^{-1}$ dispersion. ${ }^{12}$ We note that our limiting magnitude of $m_{\mathrm{F} 606 \mathrm{~W}}=23$ corresponds to $\sigma \approx 70 \mathrm{~km} \mathrm{~s}^{-1}$, and hence to

\footnotetext{
12 This assumes that the dPIE parameter $\sigma_{0}$ is a good proxy for the measured velocity dispersion, as projected and averaged over an aperture, which is true for our small $r_{\text {core }}$ (see Elíasdóttir et al. 2007).
}

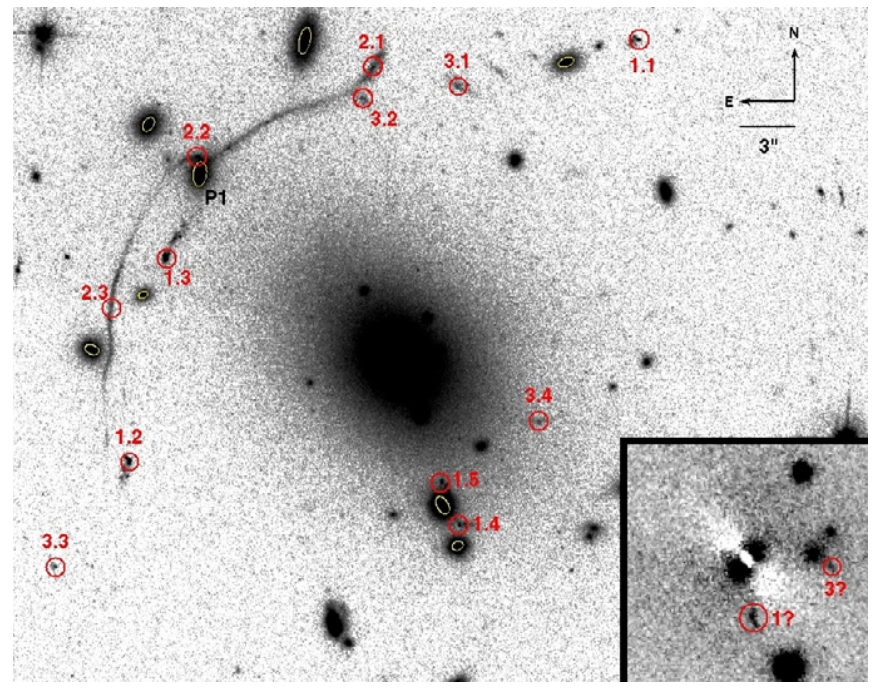

Figure 6. HST/ACS imaging of the cluster core (scaled logarithmically) and multiple image interpretation. Three multiply imaged sources are identified by the first number in each image label, with decimals distinguishing separate images. Red circles have radii indicating the positional error from the best fitting model, $\sigma_{\text {pos }}=0.5$. Cluster galaxies outlined in yellow are potentially significant lensing perturbers, and the effect of freeing them from the scaling relations was investigated (see Section 3.4); this improved the fit only for the galaxy marked P1. Inset: BCG core with the $R^{1 / 4}$ model subtracted to reveal two additional likely radial counter images used to verify the model.

a deflection angle of $\sim 0$.'07 in the singular isothermal sphere (SIS) approximation, well below our positional uncertainty of $\sigma_{\text {pos }}=0.5$.

The halo size of cluster members is uncertain, particularly in cluster cores where tidal stripping may be very efficient. Natarajan et al. (2009) found that $r_{\mathrm{cut}, *}=45 \pm 5 \mathrm{kpc}(3 \sigma)$ in the core of $\mathrm{Cl} 0024+16$ from studies of galaxy-galaxy lensing. We therefore use the scaling relation $L / L_{*}=\left(r_{\mathrm{cut}} / r_{\mathrm{cut}, *}\right)^{2}$, but impose a wide uniform prior on $r_{\mathrm{cut}, *}(30-60 \mathrm{kpc})$. Similarly, $r_{\text {core }}$ is taken to scale as $L^{1 / 2}$, but $r_{\text {core,* }}$ is fixed at $0.15 \mathrm{kpc}$, as this has a small effect.

Several cluster galaxies are quite close to images used as strong lensing constraints. It is natural to wonder whether the fit may be improved by modeling them separately. We have investigated freeing the 8 perturbers outlined in Figure 6 from the scaling laws by allowing their $\sigma_{0}$ and $r_{\text {cut }}$ parameters to vary separately (one perturber at a time), and in no case were the predicted image positions significantly improved.

We therefore judge the scaling relations sufficient to model the cluster galaxy population in the fit to strong lensing data. However, when tension from kinematic data is introduced, the giant arc is better fitted by freeing perturber P1 (Figure 6) from the scaling laws. For consistency, we optimize P1 in the strong lensing fit as well. For this perturber, $r_{\text {cut }}$ is fixed by scaling from $r_{\text {cut }, *}=45 \mathrm{kpc}$ (Natarajan et al. 2009), and $\sigma_{0}$ is allowed to vary based on the Bernardi et al. (2003) relation, with a $50 \mathrm{~km} \mathrm{~s}^{-1}$ dispersion reflecting both the intrinsic scatter and the additional uncertainty arising from fixing $r_{\text {cut }}$. It is not necessary to free both $r_{\text {cut }}$ and $\sigma_{0}$, since their effects are degenerate.

\subsection{Strong Lens Modeling of Abell 611}

Although image positions can in principle be determined very precisely from $H S T$ imaging, modeling errors dominate in practice. These include unmodeled substructure and use of functional forms (e.g., (g) NFW) that may be inappropriate 
to describe the halo. The uncertainity $\sigma_{\text {pos }}$ in image positions must therefore be inflated from the astrometric error. This uncertainty also controls the relative weight of strong lensing in the combined analysis. We find our best-fitting models are unable to reproduce image positions to better than 0.37 , and have chosen to adopt $\sigma_{\text {pos }}=0$ '.5. Smaller values of $\sigma_{\text {pos }}$ would only strengthen our claim that NFW cannot simultaneously fit all of our mass probes.

Our mass model consists of several components (relevant parameters follow in parentheses): a pseudoelliptical (g) NFW model of the DM halo $\left(\epsilon, \theta, r_{s}, \sigma_{0, \mathrm{DM}}, \beta\right)$, a dPIE model of the stellar mass in the BCG $\left(\sigma_{0, B C G}\right)$, and dPIE models of the cluster galaxy perturbers described in Section $3.4\left(r_{\mathrm{cut}, *}, \sigma_{0 *}, \sigma_{0, \mathrm{P} 1}\right)$. Since the BCG structural parameters were measured in Section 3.2, the only free parameter is $\sigma_{0, \mathrm{BCG}}$, or equivalently $M_{*} / L=1.5 \pi \sigma_{0, \mathrm{BCG}}^{2} r_{\text {cut }} / G L$ (Sand et al. 2008; Elíasdóttir et al. 2007). Note that we model only the stellar mass in the BCG, and thus identify the DM halo of the BCG with that of the cluster (see Miralda-Escude 1995). Our models of perturbing cluster members, on the other hand, represent the total mass-dark and baryonic-since there is good evidence that these galaxies maintain individual halos as they fall inward (Natarajan et al. 2009). We assume the DM halo is centered on the BCG centroid, based on our weak lensing results and the coincidence of the Chandra (ID: 3194, PI: Allen) X-ray peak within the $\sim 1 \sigma$ astrometric uncertainty. Freeing the DM center in our strong-lensing fits results in a subarcsecond offset of $\lesssim 3 \mathrm{kpc}$ but reduced Bayesian evidence. The unknown redshift of source 3 is also inferred with a uniform prior [1.5, 2.5] based on initial test runs.

We now have 11 free parameters, of which 4 are constrained by physically motivated priors, and 18 constraints. For each set of model parameters, LENSTOOL computes the image plane position $\mathbf{r}^{i}$ of each image $i$, and then

$$
\chi_{\mathrm{SL}}^{2}=\sum_{i}\left|\frac{\mathbf{r}_{i, \text { meas }}-\mathbf{r}_{i, \text { model }}}{\sigma_{\text {pos }}}\right|^{2} .
$$

Only image positions, not their fluxes or shapes, are used as constraints.

The (g) NFW mass models inferred from strong lensing constraints only are given in Table 4 . These one-dimensional marginalizations must be interpreted with caution, since severe correlations exist. These will be explored in Section 5, where the results from our three mass probes are compared. We find that both NFW and gNFW models fit the strong lensing data acceptably, with $\chi_{\mathrm{SL}}^{2}=9.5$ and 6.7 , respectively, for the maximum likelihood models, corresponding to rms position errors of 0.37 and 0.44 . A shallow inner slope $\beta=0.44_{-0.24}^{+0.40}$ is marginally preferred, but the additional complexity of the gNFW models is not demanded by the strong lensing data alone, as reflected in the evidence ratio of unity.

\subsection{Strong and Weak Lensing Combined}

At this point, we can assess whether the constraints from strong and weak lensing are compatible. There are two reasons for such a comparison. First, we compare the mass enclosed within $100 \mathrm{kpc}$, approximately the boundary between the strong and weak lensing data, to determine whether the lensing data are consistent where they (nearly) overlap. Since $M_{3 \mathrm{D}, \mathrm{SL}}$ $(<100 \mathrm{kpc})=3.04_{-0.16}^{+0.06} \times 10^{13} M_{\odot}$ and $M_{3 \mathrm{D}, \mathrm{WL}}(<100 \mathrm{kpc})=$ $2.3_{-0.5}^{+0.9} \times 10^{13} M_{\odot}$ differ by $<1 \sigma$, we see no sign of a significant discrepancy.
Table 4

(g) NFW Fits to Strong Lensing Data

\begin{tabular}{|c|c|c|c|c|}
\hline$\underline{\text { Parameter }}$ & Units & Prior & NFW Posterior & gNFW Posterior \\
\hline \multicolumn{5}{|c|}{ (g) NFW DM halo } \\
\hline$\epsilon$ & $\ldots$ & {$[0.1,0.3]$} & $0.219_{-0.014}^{+0.019}$ & $0.220 \pm 0.018$ \\
\hline P.A. & deg & {$[40,48]$} & $42.7 \pm 0.7$ & $42.7 \pm 0.7$ \\
\hline$r_{s}$ & $\mathrm{kpc}$ & {$[50,800]$} & $136_{-17}^{+24}$ & $68_{-11}^{+41}$ \\
\hline$\sigma_{0, \mathrm{DM}}$ & $\mathrm{km} \mathrm{s}^{-1}$ & {$[1000,2200]$} & $1256 \pm 30$ & $1448_{-145}^{+117}$ \\
\hline$\beta$ & $\cdots$ & {$[0.05,1.5]$} & $\ldots$ & $0.44_{-0.24}^{+0.40}$ \\
\hline$M_{200}$ & $10^{14} M_{\odot}$ & $\ldots$ & $3.50_{-0.41}^{+0.52}$ & $3.06_{-0.72}^{+0.24}$ \\
\hline$c_{200}$ & $\ldots$ & $\cdots$ & $10.0 \pm 1.1$ & $\ldots$ \\
\hline \multicolumn{5}{|c|}{ dPIE model of BCG stars } \\
\hline$\sigma_{0, \mathrm{BCG}}$ & $\mathrm{km} \mathrm{s}^{-1}$ & {$[109,345]$} & $<216$ & $<239$ \\
\hline$M_{*} / L_{B}$ & solar & $\ldots$ & $<4.0$ & $<4.8$ \\
\hline \multicolumn{5}{|c|}{ Cluster galaxy perturbers } \\
\hline$\sigma_{0 *}$ & $\mathrm{~km} \mathrm{~s}^{-1}$ & $(\mu, \sigma)=(158,50)$ & $185 \pm 18$ & $179 \pm 19$ \\
\hline$r_{\mathrm{cut}, *}$ & $\mathrm{kpc}$ & {$[30,60]$} & $<43$ & $<45$ \\
\hline$\sigma_{0, \mathrm{P} 1}$ & $\mathrm{~km} \mathrm{~s}^{-1}$ & $(\mu, \sigma)=(112,50)$ & $142_{-24}^{+18}$ & $146_{-24}^{+17}$ \\
\hline \multicolumn{2}{|c|}{ Source 3 redshift } & {$[1.5,2.5]$} & $2.11_{-0.14}^{+0.21}$ & $2.18 \pm 0.17$ \\
\hline \multicolumn{2}{|c|}{ Minimum $\chi_{\mathrm{SL}}^{2}$} & $\cdots$ & 9.5 & 6.7 \\
\hline \multicolumn{3}{|c|}{ Number of constraints (10 parameters) } & \multicolumn{2}{|c|}{18} \\
\hline Evidence $\mathrm{r}$ & atio & $\cdots$ & 1 & $1.1 \pm 0.4$ \\
\hline
\end{tabular}

Notes. Brackets indicate a uniform prior, while $(\mu, \sigma)$ indicates a Gaussian prior. Quantities without priors are derived, not directly inferred. Priors on $\epsilon$, P.A., $\sigma_{0, \mathrm{DM}}$, and the source 3 redshift were determined from initial test runs; that on $\sigma_{0, \mathrm{BCG}}$ corresponds to $1<M_{*} / L_{B}<10$. The inferred pseudoellipticity $\epsilon$ implies $b / a \approx 0.6$ for the mass surface density. Limits on $M_{*} / L_{B}$ reflect the dominant uncertainty in $M_{*}$ only, not in $L_{B}$. Error bars and upper limits refer to the $68 \% \mathrm{CL}$

Second, we compare the mass enclosed within $1.5 \mathrm{Mpc}$, approximately the virial radius inferred from weak lensing, to determine if the $(\mathrm{g}) \mathrm{NFW}$ profiles adequately describe both lensing regimes simultaneously. This is directly constrained from weak lensing to be $M_{3 \mathrm{D}, \mathrm{WL}}(<1.5 \mathrm{Mpc})=(7.4 \pm 1.4) \times$ $10^{14} M_{\odot}$, and is extrapolated from the strong lensing results to be $M_{3 \mathrm{D}, \mathrm{SL}}(<1.5 \mathrm{Mpc})=(3.70 \pm 0.41) \times 10^{14} M_{\odot}$ using an NFW profile. (Adopting a gNFW model instead shifts these by $<1 \sigma$.) The significance of this difference $(\sim 2.5 \sigma)$ suggests marginal tension in the ability of a $(\mathrm{g})$ NFW profile to fit all the lensing data simultaneously, but is not conclusive in itself.

The lensing data may be formally combined by setting $\chi^{2}=\chi_{\mathrm{SL}}^{2}+\chi_{\mathrm{WL}}^{2}$. As expected, little information on the inner slope is obtained: the posterior probability density is flat over a wide range ( $\beta \sim 0.6-1.5)$ and falls off slowly. Correspondingly, the evidence ratio NFW $: \mathrm{gNFW}=(2.2 \pm 0.6): 1$ is near unity.

\section{STELLAR KINEMATICS AND DYNAMICAL ANALYSIS}

\subsection{Observations and Data Reduction}

We observed the Abell 611 BCG on 2008 March 3 using the long-slit mode on the LRIS spectrograph at the Keck I telescope, using a $600 \mathrm{~mm}^{-1}$ grating blazed at $7500 \AA$, targeting the redshifted Fe lines around $6800 \AA$. The $1^{\prime \prime} .5$ wide slit was aligned within $3^{\circ}$ of the major axis, as measured in Section 3.2. The average seeing was $\sim 1^{\prime \prime}$. 4 . Five exposures were taken for 130 minutes total integration.

The LRIS data were reduced in a standard manner with bias subtraction, flat-fielding via quartz lamp exposures taken between science observations, and cosmic-ray rejection using LACOSMIC (van Dokkum 2001). Spatial and spectral distortions were removed with the IRAF tasks IDENTIFY, REIDENTIFY, 


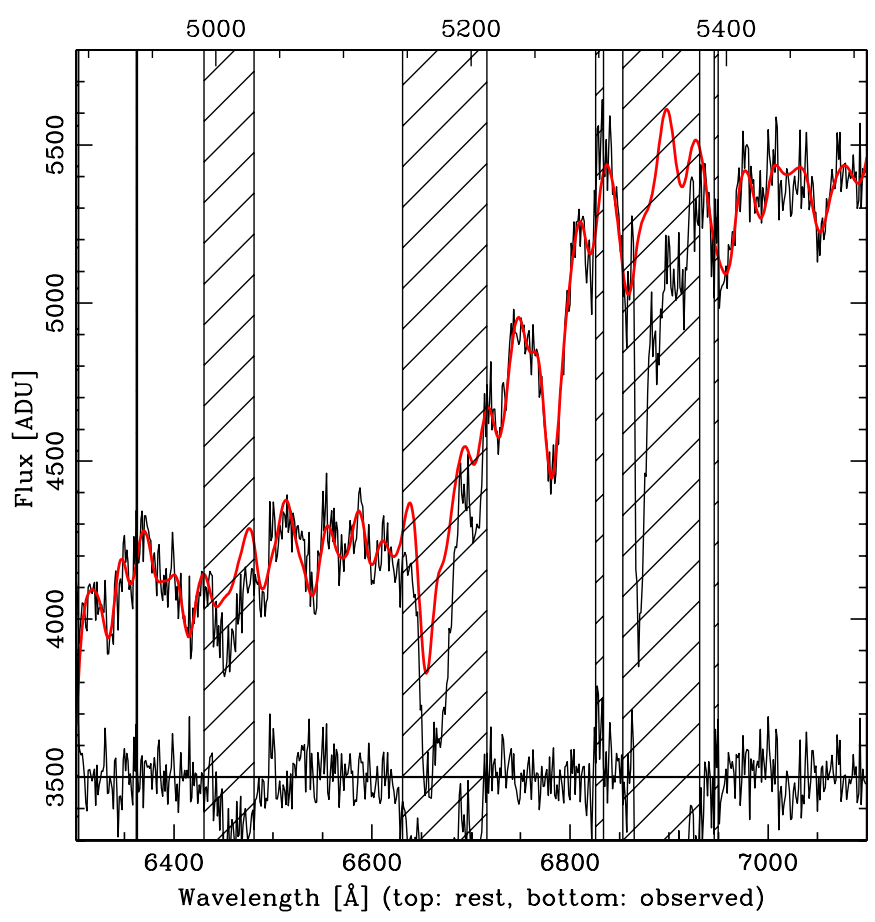

Figure 7. Central spatial bin of the BCG spectrum (black) compared with the best-fitting model described in the text (red), with residuals shown below. The atmospheric B-band around $6900 \AA$ is excluded from the fit, as are narrow bright sky lines. Also masked are two poorly fit spectral lines, including $\mathrm{Mg} b$. As discussed by Barth et al. (2002), it is not possible to simultaneously fit $\mathrm{Mg} b$ and the $\mathrm{Fe}$ lines, since $[\mathrm{Mg} / \mathrm{Fe}]$ is itself a strong function of the velocity dispersion.

(A color version of this figure is available in the online journal.)

FITCOORDS, and TRANSFORM. Wavelength calibration was determined directly from night sky lines, with residuals of order $0.2 \AA$. The slit function was determined from night sky lines, which were then subtracted by low-order fitting. The spectral resolution was measured from unblended night sky lines to be $\sigma=99 \mathrm{~km} \mathrm{~s}^{-1}$ over the spectral range used for velocity dispersion measurement (6300-7100 $\AA$ ). The spectra were spatially binned to provide sufficient $S / N(\gtrsim 20$ per pixel) for a reliable determination. Bins were also required to contain at least 5 CCD rows, comparable to the seeing element, to avoid an excess of correlated points. One bin was excluded due to possible contamination from an interloping galaxy.

\subsection{Velocity Dispersion Measurements}

Velocity dispersions were measured using a direct fitting procedure similar to that of Sand et al. (2004, 2008). Highresolution template spectra of G0III-K2III giants, previously obtained using ESI on the Keck II telescope, were convolved with a Gaussian to match the LRIS instrumental resolution. The templates were then redshifted and rebinned to match the range (6300-7100 $\AA$ ) and dispersion $\left(57 \mathrm{~km} \mathrm{~s}^{-1}\right.$ pixel $\left.^{-1}\right)$ of the BCG data. Model galaxy spectra were computed assuming a Gaussian line-of-sight velocity distribution (LOSVD). We fit these models to the data with a modified version of the code described by van der Marel (1994). The best-fitting model is that which minimizes

$$
\chi^{2}=\sum_{i}\left(\frac{S_{i}-\left[P_{M}\left(\lambda_{i}\right) \cdot\left(B_{\sigma_{\mathrm{LOS}}, z} \circ T\right)\left(\lambda_{i}\right)+P_{N}\left(\lambda_{i}\right)\right]}{\sigma_{i}}\right)^{2},
$$

Table 5

BCG Stellar Velocity Dispersions

\begin{tabular}{|c|c|c|c|}
\hline Spatial Bin & $(\mathrm{kpc})$ & $\langle\mathrm{S} / \mathrm{N}\rangle /$ Pixel & $\sigma_{\mathrm{LOS}}\left(\mathrm{km} \mathrm{s}^{-1}\right)$ \\
\hline$-5^{\prime \prime} .2$ to $-3^{\prime \prime} .7$ & -22 to -16 & 21 & $316 \pm 67$ \\
\hline-3 ". 7 to $-2^{\prime \prime} .6$ & -16 to -11 & 27 & $308 \pm 40$ \\
\hline$-2^{\prime \prime} .6$ to $-1^{\prime \prime} .6$ & -11 to -4.2 & 39 & $330 \pm 28$ \\
\hline$-1^{\prime \prime} .6$ to $-0^{\prime \prime} .5$ & -4.2 to -2.2 & 58 & $333 \pm 18$ \\
\hline$-0^{\prime \prime} 5$ to 0.5 & -2.2 to 2.2 & 73 & $287 \pm 15$ \\
\hline $0^{\prime \prime} 5$ to $1^{\prime \prime} .6$ & 2.2 to 4.2 & 58 & $293 \pm 17$ \\
\hline $1^{\prime \prime} .6$ to $2^{\prime \prime} .6$ & 4.2 to 11.3 & 38 & $308 \pm 25$ \\
\hline $2^{\prime \prime} .6$ to $3^{\prime \prime} .7$ & 11 to 16 & 25 & $\ldots$ \\
\hline $33^{\prime \prime} 7$ to $5^{\prime \prime} .4$ & 16 to 23 & 21 & $344 \pm 40$ \\
\hline
\end{tabular}

Notes. Negative (positive) positions are southwest (northeast) of the centroid along the slit. Errors are $1 \sigma$ statistical plus a systematic estimate $\left(10 \mathrm{~km} \mathrm{~s}^{-1}\right)$ added in quadrature. The $11-16 \mathrm{kpc}$ bin is excluded due to possible contamination from an interloper. The dispersion is $1.3 \AA$ pixel $^{-1}$.

where $S_{i}$ is the measured spectrum, $i$ enumerates the pixels, $B_{\sigma_{\mathrm{LOS}, z}} \circ T$ is the broadened and redshifted template, $P_{M}(\lambda)$ and $P_{N}(\lambda)$ are $M$ and $N$ order polynomials, respectively, and $\sigma_{i}$ is the flux uncertainty computed from standard CCD parameters. The free parameters are $\sigma_{\mathrm{LOS}}$, redshift, and coefficients of $P_{N}$ and $P_{M} . P_{N}$ is an additive term used to model the galaxy continuum. $P_{M}$ is a multiplicative term containing the normalization and any wavelength-dependent differences in instrument response between the galaxy and template spectra.

Given the spectral range of $800 \AA$, we anticipate that $N \approx 9$ is necessary to model continuum variations on $100 \AA$ scales. Low $N$ will cause a poor fit and a biased measurement, while high $N$ allow the polynomial to fit spectral lines. Indeed, $N=9$ provides a significantly better fit than $N=8$, while for higher $N$ the fit quality plateaus. We therefore take $N=9$ and adopt a multiplicative order $M=2$ to model any uncorrected differences in instrument response. The best fit was obtained with the G9III template, and the central spatial bin is shown in Figure 7. Spectral regions around bright sky lines, the atmospheric B-band absorption, and two poorly fit lines were excluded. The fit is excellent, particularly around the dominant Fe $\lambda 5270$ line, and the small, pattern-free residuals indicate no serious systematic effects.

Measured dispersions for all bins are given in Table 5. The data are statistically consistent with a flat dispersion profile out to our radial limit. Rotation was not detected and must be $\lesssim 20 \mathrm{~km} \mathrm{~s}^{-1}$, constituting $\lesssim 0.5 \%$ of the kinetic energy.

To evaluate systematic errors in our fitting procedure (due, e.g., to template mismatch or poor continuum fitting), four different spectral regions were fit using various continuum orders and stellar templates (G7III-K0III), and the systematic uncertainty was estimated from the scatter in derived velocity dispersions to be $\approx 10 \mathrm{~km} \mathrm{~s}^{-1}$. We add this in quadrature to the statistical errors derived from the $\chi^{2}$ surface to produce the estimates in Table 5. The effect of systematic changes to the velocity dispersions on our results is discussed in Section 5.2.

\subsection{Dynamical Modeling}

We have incorporated velocity dispersion constraints into LENSTOOL. Each model suggested by the MCMC sampler specifies the DM halo parameters and the stellar $M_{*} / L$. Projected stellar velocity dispersions $V_{*}$ are then computed based on the (g) NFW halo and the $R^{1 / 4}$ model of the BCG (Section 3.2). Spherical symmetry and isotropic orbits are assumed here, as 
Table 6

(g) NFW Fits to Strong Lensing + Kinematic Data

\begin{tabular}{|c|c|c|c|c|}
\hline Parameter & Units & Prior & NFW Posterior & gNFW Posterior \\
\hline \multicolumn{5}{|l|}{ (g) NFW DM halo } \\
\hline$\epsilon$ & $\ldots$ & {$[0.1,0.3]$} & $0.165 \pm 0.008$ & $0.179 \pm 0.009$ \\
\hline P.A. & deg & {$[40,48]$} & $43.0 \pm 0.8$ & $42.8 \pm 0.7$ \\
\hline$r_{s}$ & $\mathrm{kpc}$ & {$[50,800]$} & $229_{-18}^{+24}$ & $65^{+11}$ \\
\hline$\sigma_{0, \mathrm{DM}}$ & $\mathrm{km} \mathrm{s}^{-1}$ & {$[1000,2200]$} & $1408 \pm 30$ & $1663_{-53}^{+36}$ \\
\hline$\beta$ & & {$[0.05,1.5]$} & & $<0.22$ \\
\hline$M_{200}$ & $10^{14} M_{\odot}$ & $\ldots$ & $6.1_{-0.5}^{+0.6}$ & $3.6_{-1.0}^{+0.3}$ \\
\hline$c_{200}$ & - & $\cdots$ & $7.05 \pm 0.42$ & \\
\hline \multicolumn{5}{|c|}{ dPIE model of BCG stars } \\
\hline$\sigma_{0, \mathrm{BCG}}$ & $\mathrm{km} \mathrm{s}^{-1}$ & {$[109,345]$} & $<123$ & $169_{-28}^{+22}$ \\
\hline$M_{*} / L_{B}$ & solar & & $<1.3$ & $2.3 \pm 0.7$ \\
\hline \multicolumn{5}{|c|}{ Cluster galaxy perturbers } \\
\hline$\sigma_{0 *}$ & $\mathrm{~km} \mathrm{~s}^{-1}$ & $(\mu, \sigma)=(158,50)$ & $167 \pm 15$ & $157 \pm 17$ \\
\hline$r_{\mathrm{cut}, *}$ & $\mathrm{kpc}$ & {$[30,60]$} & $\ldots$ & $\ldots$ \\
\hline$\sigma_{0, \mathrm{P} 1}$ & $\mathrm{~km} \mathrm{~s}^{-1}$ & $(\mu, \sigma)=(112,50)$ & $169 \pm 16$ & $162_{-17}^{+13}$ \\
\hline Source 3 redshift & $\cdots$ & {$[1.5,2.5]$} & $1.91 \pm 0.11$ & $2.03_{-0.11}^{+0.16}$ \\
\hline Minimum $\chi_{\mathrm{SL}}^{2} / \chi_{\mathrm{VD}}^{2}$ & $\ldots$ & $\ldots$ & $31.8 / 51.0$ & $11.3 / 41.8$ \\
\hline \multicolumn{3}{|c|}{ Number of constraints ( 10 parameters) } & \multicolumn{2}{|r|}{$18 / 8$} \\
\hline Evidence ratio & $\ldots$ & $\ldots$ & 1 & $(1.9 \pm 0.6) \times 10^{5}$ \\
\hline
\end{tabular}

Note. See notes to Table 4 . Since $r_{\text {cut,*}}$ was essentially unconstrained, we omit a measure of its (flat) posterior.

discussed extensively in Section 5.2, and we therefore circularize the bin radii in our data. The dispersion and deprojected density profiles for the $R^{1 / 4}$ law are given by Young (1976). The effects of seeing, slit width, and spatial binning were accounted for as described in Sand et al. (2004), and

$$
\chi_{\mathrm{VD}}^{2}=\sum_{i}\left(\frac{V_{* i, \mathrm{obs}}-V_{* i, \text { model }}}{\sigma_{i}}\right)^{2}
$$

was computed by summing over the 8 bins in Table 5 .

Although these kinematic data alone cannot uniquely constrain the mass model, they acquire great power to determine the mass profile at $\lesssim 20 \mathrm{kpc}$ scales when combined with the strong lensing data. We coupled the kinematic and strong lensing data by setting $\chi^{2}=\chi_{\mathrm{SL}}^{2}+\chi_{\mathrm{VD}}^{2}$. In total, this provides 26 constraints on the same (g) NFW models considered in Section 3.5. The inferred parameters are given in Table 6. Figure 8 presents the velocity dispersion profiles derived from NFW and gNFW models to these data, as well as those inferred from strong lensing data alone (Section 3.5).

There are several important points to note. First, an NFW extrapolation of strong-lensing constraints on $\sim 30-90 \mathrm{kpc}$ scales overestimates the mass at radii $\lesssim 20 \mathrm{kpc}$, and hence the central velocity dispersion. Second, an NFW fit can more nearly match the kinematic data when it too is imposed as a constraint, but the quality of fit at larger radii then suffers dramatically $\left(\Delta \chi_{\mathrm{SL}}^{2}=22\right)$. The constraints on both scales cannot be simultaneously met. Third, when the asymptotic inner slope $\beta$ is freed by adopting the gNFW profile, a similar fit to the kinematic data is obtained, while incurring far less discrepancy with the strong lensing data $\left(\Delta \chi_{\mathrm{SL}}^{2}=5\right)$. This is achieved by selecting $\beta<0.22(68 \%)$, a much shallower density profile than NFW. As discussed in Sand et al. (2004), only upper limits on $\beta$ are available for systems without a radial arc. Although we identified potential radial arcs in Section 3.3, they have not been used to constrain our models, due to their uncertain identification. We therefore expect our inference of $\beta$ to be one sided.

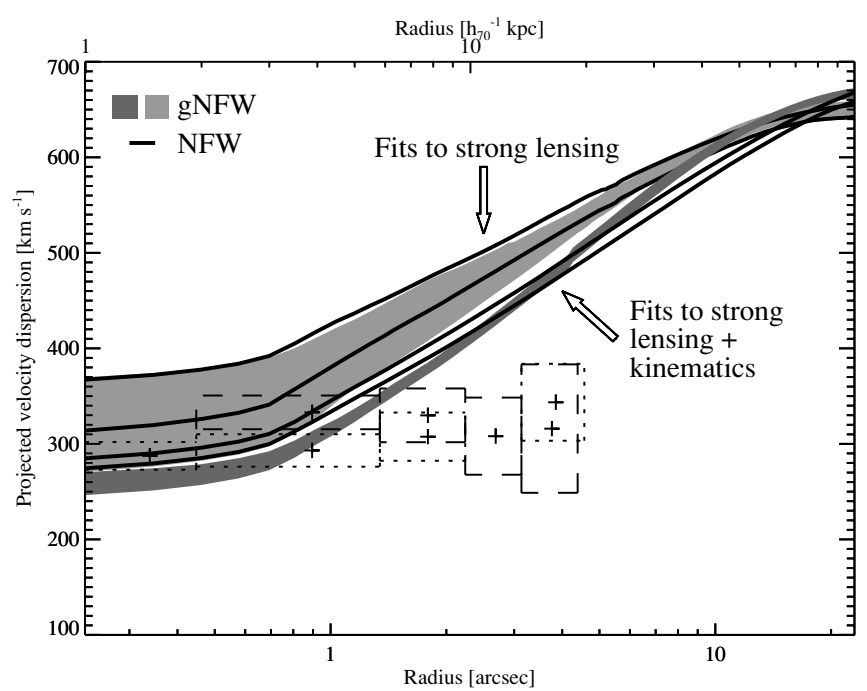

Figure 8. Measured stellar velocity dispersions (rectangles) with NFW (lines) and gNFW (shaded) fits to strong lensing data alone (upper) and to strong lensing and kinematic data (lower). A NFW extrapolation from larger scales overpredicts the velocity dispersion. Models have been PSF-convolved to match the data. For the data, radii have been circularized from their major axis positions by multiplying by $(b / a)^{1 / 2}$. The dotted and dashed rectangles distinguish measurements on either side of the center. The $68 \%$ confidence regions are shown.

\section{JOINT ANALYSIS OF THE DARK MATTER DISTRIBUTION: 3 KPC-3.25 MPC}

In this section, we now realize our goal of combining the three observational techniques: weak lensing from $150 \mathrm{kpc}$ to $3 \mathrm{Mpc}$, strong lensing from $\sim 30-90 \mathrm{kpc}$, and stellar kinematics from $\sim 3-20 \mathrm{kpc}$, in order to probe the dark matter distribution, independently of the baryonic contribution. The wide dynamic range of our data provides a stringent test of theory. We seek to accomplish two goals: (1) to verify or otherwise the validity of the NFW profile for Abell 611, (2) in the specific case of the gNFW profile, to determine the likely range of the variable 
inner slope $\beta$. In achieving these aims, we will also demonstrate the value of our approach. Specifically, we demonstrate that although an acceptable fit to the NFW profile might be achieved with a subset of our data over a more limited radial extent, such an agreement is illusory when the broader range of data is taken into account.

We begin by considering Figure 9(a), which shows the mass profile obtained from NFW fits to our three data sets. The weak lensing data is well fit by an NFW profile, with a concentration appropriate to its mass and in excellent agreement with X-ray data, as discussed in Section 2.5. By looking at large scales only, we would therefore confirm CDM predictions. On intermediate scales, we found that positions of multiply imaged sources could also be reproduced to good precision by models including an NFW halo. However, this fit diverges from that obtained via weak lensing (and X-ray) data on Mpc scales, as indicated in Figure 9(a).

The most serious discrepancy comes on $\lesssim 20 \mathrm{kpc}$ scales, where we find NFW extrapolations from the strong lensing regime imply velocity dispersions significantly higher than are measured. The dispersion data can be partially matched only by incurring drastically increased errors in the predicted positions of strongly lensed sources. (Since strong lensing constraints are the most precise, even strong deviations are not visible on the scale of Figure 9. The severity of the problem was discussed in Section 3.5.)

We have also considered a simple generalization of the NFW profile, motivated by controversy in the theoretical literature regarding the inner form of the DM distribution. By freeing the inner slope $\beta$, gNFW models have the extra freedom to decrease the central mass as the kinematic data require, while incurring less damage to the fit quality on larger scales. We found only marginal evidence for a flat slope $\beta<0.7(68 \%)$ in fits to strong lensing data alone. Including kinematic data in the inner $20 \mathrm{kpc}$ forced a flat slope with high confidence: $\beta<0.2(68 \%)$. Finally, we have formally combined our three data sets by setting

$$
\chi^{2}=\chi_{\mathrm{WL}}^{2}+\chi_{\mathrm{SL}}^{2}+\chi_{\mathrm{VD}}^{2}
$$

and sampling with Lenstool. Posterior distributions are listed in Table 7. The effect is to slightly lower the evidence in favor of the gNFW profile, since the NFW fit to strong lensing + kinematic data, when extrapolated to large scales, agrees somewhat better with the weak lensing data than does the gNFW fit. (This can be seen in Figure 9.)

The Bayesian evidence overwhelmingly favors the gNFW model over the NFW model, by a factor of $2 \times 10^{4}$. This corresponds to a preference significant at more than $99 \%$. Correspondingly, a shallow inner slope is strongly preferred, with $\beta<0.56$ at the $95 \% \mathrm{CL}(\beta<0.65,99 \% \mathrm{CL})$. The inner slope is degenerate with the scale radius and the $M_{*} / L$ ratio of the baryons, as shown in Figure 10, but we cannot reconcile $\beta$ with an NFW-like slope if the scale radius is to be compatible with the data, and the $M_{*} / L$ ratio consistent with stellar evolution theory. Although recent numerical work (e.g., Navarro et al. 2008) has suggested that slopes shallower than NFW may be found just beyond current limit of numerical resolution, the difference may be insufficient to explain the data. Figure 11 demonstrates this by showing that on the scales we probe, the Einasto profile, another functional form fit to $\mathrm{N}$-body results (e.g., Navarro et al. 2004), is much more similar to the NFW form than either is to the gNFW models we infer.

Although the gNFW profile provides a simple alternative that our data support decisively over NFW, it too cannot be
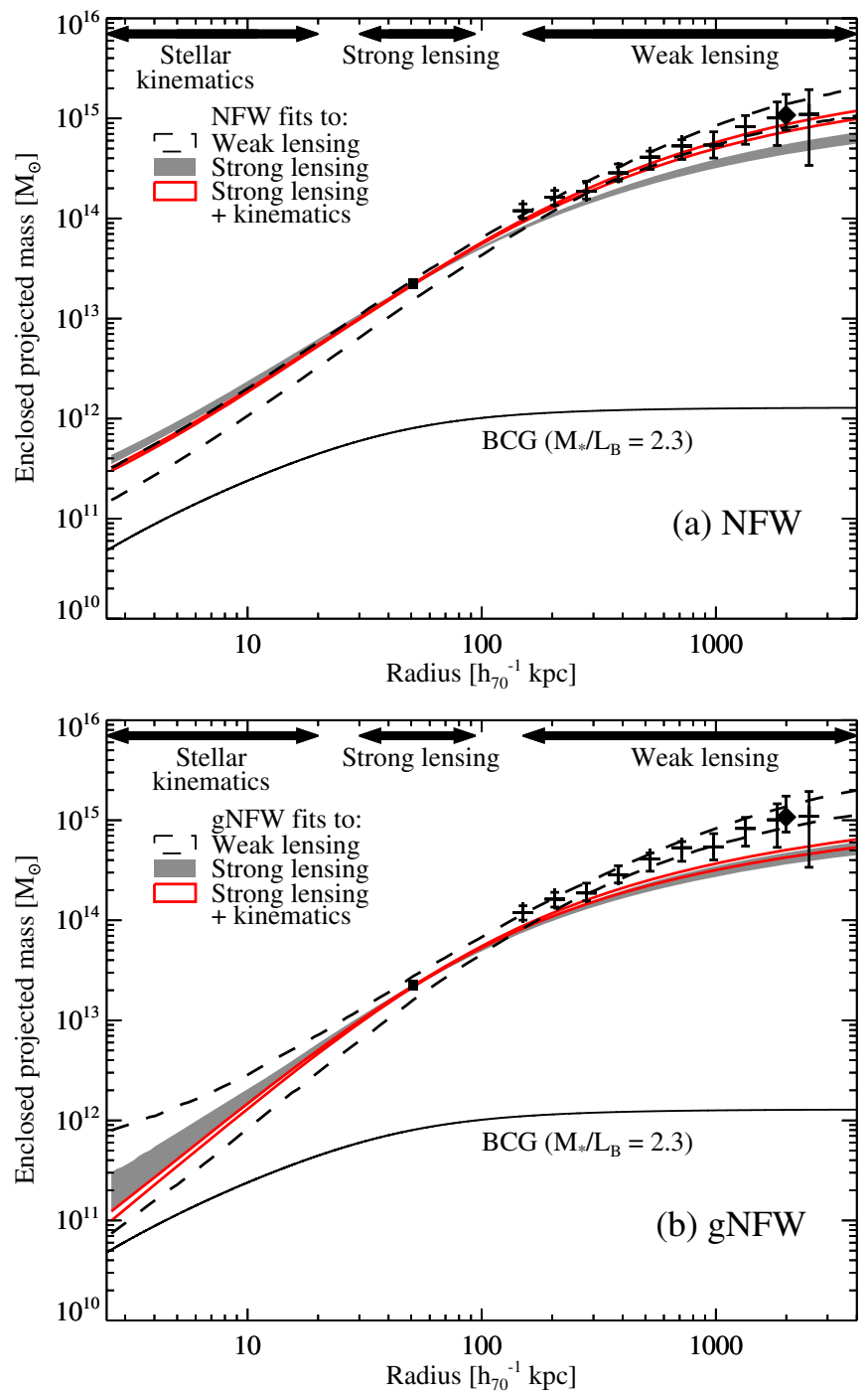

Figure 9. (a) Enclosed projected two-dimensional mass inferred from NFW fits to our three data sets, with curves bracketing the $68 \%$ confidence region. Note that a single NFW profile is unable to match the dark matter distribution at all radii; instead, the NFW-based extrapolations of data are discrepant on kpc and Mpc scales. The filled box gives the location of the giant tangential arc. For reference, crosses denote weak-lensing circular aperture masses obtained from the $\zeta_{c}$ statistic (Clowe et al. 1998) (68\%). Note that these points are correlated. The filled diamond denotes the X-ray mass at the virial radius (Schmidt \& Allen 2007) (68\%). The stellar contribution of the BCG is shown for the $M_{*} / L_{\mathrm{B}}$ of Table 6. Lensing fits are fully elliptical, and the radius plotted here is the elliptical radius. (b) Same, except using gNFW models. Note that the additional flexibility of the gNFW profile permits less mass on $\lesssim 10 \mathrm{kpc}$ scales.

(A color version of this figure is available in the online journal.)

considered a fully satisfactory description of the data. Tension with the strong lensing data remains (Section 3.5), and the observed flatness of the dispersion data (Figure 8) is not reproduced. The shape of this profile is likely affected by the poorly understood ways in which baryons shape DM in cluster cores, which we discuss in Section 6.

Finally, motivated by some theoretical suggestions that the NFW profile may in fact better describe the total matter distribution (e.g., Gao et al. 2004), we have constructed the total density profile by adding the dark and stellar components of our combined gNFW models. This total density profile is indeed intriguingly closer to NFW. However, we note that when comparing fits to our two-component gNFW+BCG model with 

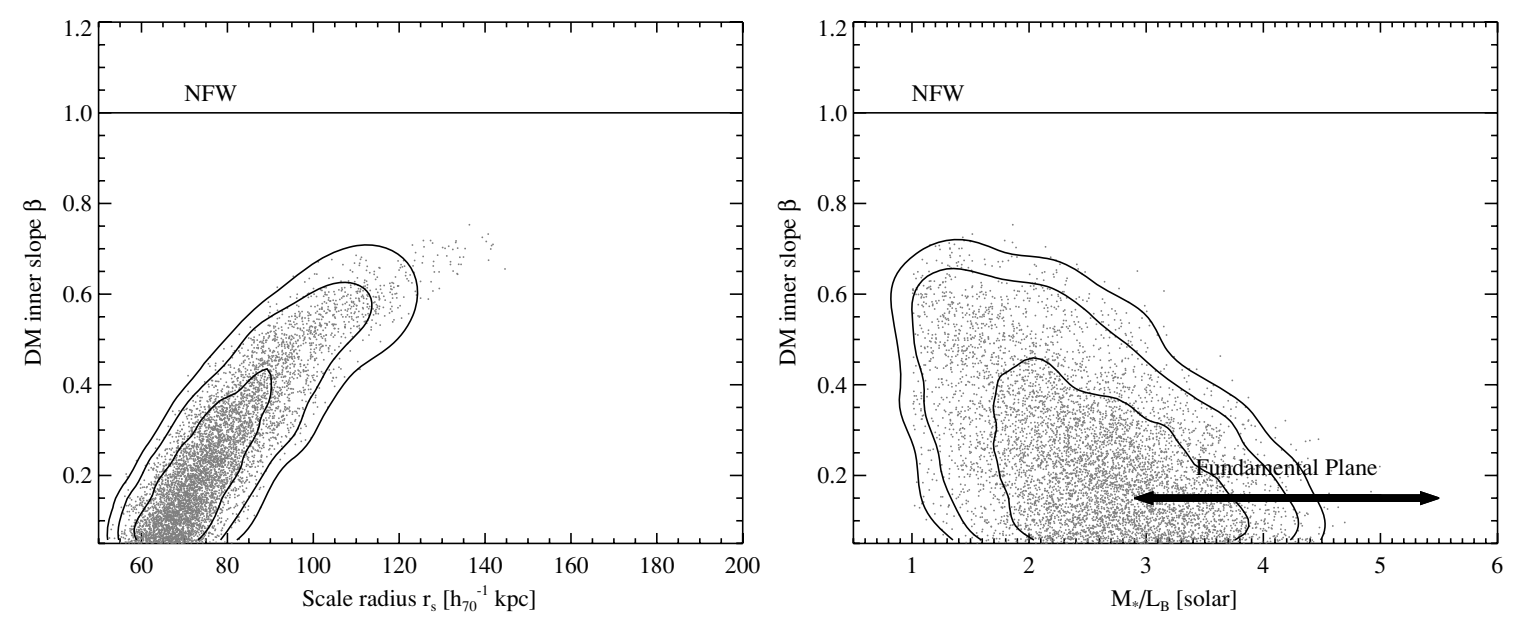

Figure 10. Left: degeneracy between DM inner slope $\beta$ and scale radius $r_{s}$. The slope is compatible with NFW only for scale radii much larger than allowed by the data. Contours indicate the $68 \%, 95 \%$, and $99 \%$ confidence regions. MCMC samples are plotted. Right: degeneracy between $\beta$ and $M_{*} / L_{B}$. Large $M_{*} / L_{B}$ ratios can mimic a shallow slope $\beta$ : they imply a more massive BCG, and hence less DM in the central region in order to maintain the same total mass. Fundamental plane constraints at this redshift from Treu \& Koopmans (2004) are shown (68\%).

Table 7

(g) NFW Fits to Strong + Weak Lensing and Kinematic Data

\begin{tabular}{|c|c|c|c|c|}
\hline Parameter & Units & Prior & NFW Posterior & gNFW Posterior \\
\hline \multicolumn{5}{|c|}{ (g) NFW DM halo } \\
\hline$\epsilon$ & $\ldots$ & {$[0.1,0.3]$} & $0.163 \pm 0.008$ & $0.173_{-0.007}^{+0.009}$ \\
\hline P.A. & deg & {$[40,48]$} & $42.9 \pm 0.8$ & $42.6_{-0.6}^{+0.801}$ \\
\hline$r_{s}$ & $\mathrm{kpc}$ & {$[50,800]$} & $236_{-18}^{+24}$ & $71_{-6}^{10.0}$ \\
\hline$\sigma_{0, \mathrm{DM}}$ & $\mathrm{km} \mathrm{s}^{-1}$ & {$[1000,2200]$} & $1415 \pm 30$ & $1682_{-81}^{+37}$ \\
\hline$\beta$ & $\ldots$ & {$[0.05,1.5]$} & $\ldots$ & $<0.30$ \\
\hline$M_{200}$ & $10^{14} M_{\odot}$ & $\cdots$ & $6.2_{-0.5}^{+0.7}$ & $3.8_{-0.6}^{+0.4}$ \\
\hline$c_{200}$ & $\ldots$ & $\cdots$ & $6.95 \pm 0.41$ & $\cdots$ \\
\hline \multicolumn{5}{|c|}{ dPIE model of BCG stars } \\
\hline$\sigma_{0, \mathrm{BCG}}$ & $\mathrm{km} \mathrm{s}^{-1}$ & {$[109,345]$} & $<123$ & $179_{-30}^{+20}$ \\
\hline$M_{*} / L_{B}$ & solar & $\cdots$ & $<1.3$ & $2.7_{-0.8}^{+0.70}$ \\
\hline \multicolumn{5}{|c|}{ Cluster galaxy perturbers } \\
\hline$\sigma_{0 *}$ & $\mathrm{~km} \mathrm{~s}^{-1}$ & $(\mu, \sigma)=(158,50)$ & $164 \pm 15$ & $145 \pm 26$ \\
\hline$r_{\mathrm{cut} *} *$ & $\mathrm{kpc}$ & {$[30,60]$} & $\ldots$ & $\ldots$ \\
\hline$\sigma_{0, \mathrm{P} 1}$ & $\mathrm{~km} \mathrm{~s}^{-1}$ & $(\mu, \sigma)=(112,50)$ & $170 \pm 16$ & $155 \pm 16$ \\
\hline \multicolumn{2}{|c|}{ Source 3 redshift } & {$[1.5,2.5]$} & $1.88_{-0.09}^{+0.13}$ & $1.99_{-0.11}^{+0.14}$ \\
\hline \multirow{2}{*}{\multicolumn{2}{|c|}{$\begin{array}{l}\text { Shear calibration } m \\
\text { Minimum } \chi_{\mathrm{SI}}^{2} / \chi_{\mathrm{VD}}^{2} / \chi_{\mathrm{W}}^{2}\end{array}$}} & $(\mu, \sigma)=(0.81,0.05)$ & $\begin{array}{l}-0.09 \\
0.85_{-0.07}^{+0.05}\end{array}$ & $0.87_{-0.06}^{-0.11}$ \\
\hline & & $\ldots$ & $31.9 / 50.8 / 13081.0$ & $16.0 / 38.2 / 13086.7$ \\
\hline \multicolumn{3}{|c|}{ Number of constraints (11 parameters) } & \multicolumn{2}{|c|}{$18 / 8 / 13090$} \\
\hline \multicolumn{3}{|c|}{ Evidence ratio } & 1 & $(2.2 \pm 1.0) \times 10^{4}$ \\
\hline
\end{tabular}

Note. See notes to Table 6.

fits to a single-component NFW model, the two-component model is still favored by a factor of $545 \pm 301$ in evidence.

Since our analysis includes a number of simplifying assumptions that could in principle bias the inferred density profile, particularly $\beta$, we have performed a battery of tests to evaluate known systematic effects, which are discussed in turn below. We find that none of these systematic effects can seriously alter our basic results, and thus believe that our findings are robust. Figure 12 shows the distribution of $\beta$ we infer from our combined fit to all data sets and summarizes the effects of the systematics discussed below. In all cases, we retain $\beta<1$ at $>98 \%$ confidence. Furthermore, the dominant systematic is likely to be the exclusion of baryons from the $N$-body simulations to which we compare data. Although the theory is insufficiently developed to make good numerical estimates, it is generally believed that including baryons will widen the discrepancy we find with simulations (see Section 6).

\subsection{Triaxiality and Projection Effects}

Dark matter halos are expected to be triaxial. In the thin-lens approximation applicable to galaxy clusters, lensing depends only on the projected surface density, which we model in a fully elliptical fashion. However, unmodeled elongation along the line of sight (los) does affect dynamical inferences, as these depend on the three-dimensional mass structure. The possible impact of triaxiality on our results can be estimated by using priors from cosmological simulations to infer the likely three-dimensional shape of the DM halo.

The halo pseudeoellipticity inferred from strong lensing (Table 4) corresponds to a projected (mass) axis ratio of $q=0.6$ (Golse \& Kneib 2002, Equation (28)). Equations relating the shape of a triaxial ellipsoid, with axis ratios $c \leqslant b \leqslant 1$, to its two-dimensional projection are given by Romanowsky \& Kochanek (1998). We used Monte Carlo sampling to find 

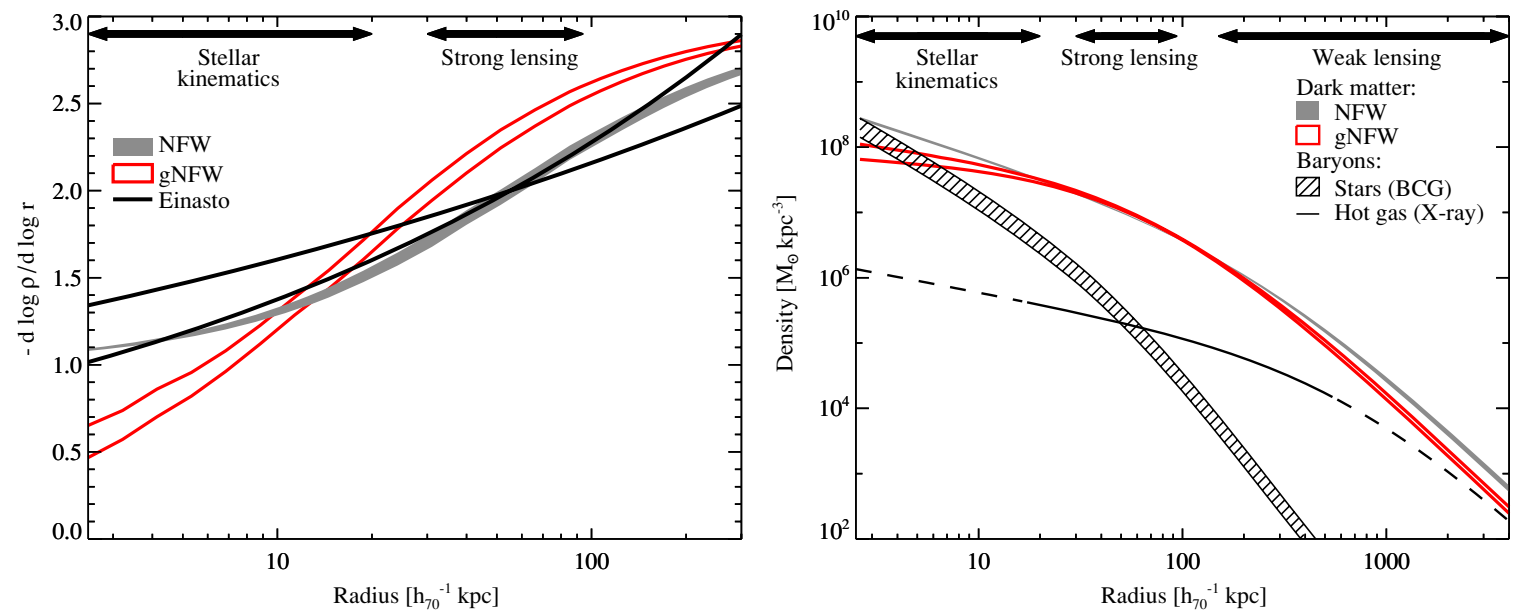

Figure 11. Left: logarithmic slope of the density profile inferred from (g) NFW fits to weak and strong lensing and stellar kinematics. For comparison, Einasto profiles with $\alpha=0.129$ and 0.219 are plotted, bracketing the range noted by Navarro et al. (2004) in cluster simulations; here $r_{-2}$ is fixed to the median $r_{s}$ inferred in NFW fits. Navarro et al. (2004) used such a profile to fit the inner regions of clusters in their $N$-body simulations. All confidence regions are $68 \%$. Right: density profiles inferred from (g) NFW fits to the combined data set. The BCG profile is also shown, using the $M_{*} / L$ ratios inferred in the gNFW model, along with the X-ray gas profile provided by S. Allen (Section 5.3). For reference, the dashed line gives a gNFW extrapolation of the hot gas outside the range of the X-ray data.

(A color version of this figure is available in the online journal.)

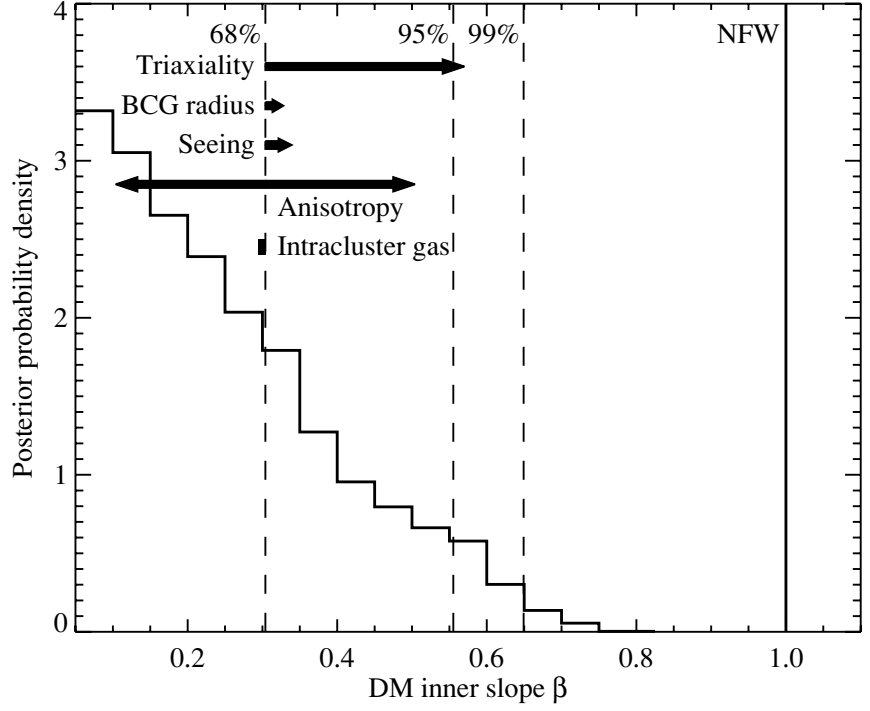

Figure 12. Marginalized posterior probability density for $\beta$, as inferred from the combined fit to weak lensing, strong lensing, and kinematic data. The effects of the systematic effects discussed in Sections 5.1-5.4 on the 68\% upper limit are illustrated by arrows.

the $b, c$, and los orientations compatible with the observed $q$ and prior distributions $p(c)$ and $p(b \mid c)$ from the $N$-body DM simulations presented by Jing \& Suto (2002), as suggested by Gavazzi (2005). We find that the radius along the los is $>0.6$ $(2 \sigma)$ of the average radius in the plane of the sky; that is, strong compression along the los is unlikely. Furthermore, interaction with baryons tends to make halos rounder and less triaxial than those in pure DM simulations (Gustafsson et al. 2006; Debattista et al. 2008; Abadi et al. 2009).

Gavazzi (2005) has estimated the error on the enclosed mass as inferred from kinematic data by assuming spherical symmetry, when the actual mass distribution is a prolate (oblate) ellipsoid aligned with major (minor) axis along the los. A prolate halo would exacerbate the discrepancy we find with NFW, since stars move faster along the major axis, and hence dynamical masses are overestimated. An oblate halo with short axis along the los causes an downward bias in dynamical mass, which is $\lesssim 30 \%$ for the above limit on $c$. This bias can be approximately compensated by increasing the velocity dispersion measurements by $15 \%$. Repeating our combined gNFW analysis with this modification yields $\beta<0.57(68 \%$; $\beta<0.92,95 \% \mathrm{CL})$.

We therefore believe our results are robust against likely projection effects, although the significances may vary. Nevertheless, recognizing that triaxiality has a complicated effect on dynamical masses, and the possible inadequacy of simple models in the present situation (e.g., the simplifying assumptions by Gavazzi 2005 of a spheroidal halo aligned with the los and massless tracers), we intend to pursue this issue more rigorously in future work, both observationally and in modeling. Additionally, a larger sample will allow us to assess the likelihood that chance alignments of triaxial halos can explain our findings.

\subsection{Velocity Dispersion Measurements and Modeling}

In our dynamical modeling, we assumed isotropic stellar orbits and a Gaussian LOSVD. Since the mass distribution inferred from a velocity dispersion profile depends on the anisotropy tensor, the effects of these assumptions must be considered. Orbital structure has been studied extensively in local samples of $\mathrm{cD}$, cluster, and field elliptical galaxies (e.g., Gerhard et al. 1998; Saglia et al. 2000; Kronawitter et al. 2000; Gerhard et al. 2001; Thomas et al. 2005, 2007, see also Kelson et al. 2002 and references), and also in a few distant early-type galaxies (Treu \& Koopmans 2004). The broad consensus is that orbital structure in the inner regions is remarkably consistent: along the major axis, orbits range from isotropic to slightly radially biased, with the isotropy parameter $\beta$ typically $\lesssim 0.3$ and up to $\approx 0.5$. Moreover, deviations from a Gaussian LOSVD are small, with $h_{3}$ and $h_{4}$ typically a few percent and (rarely) up to $10 \%$. At the radii we probe $\left(\lesssim 0.4 R_{e}\right)$, radial bias causes the projected velocity dispersion to be higher than would be seen for isotropic orbits in the same mass distribution (Binney \& Tremaine 1987, Figure 4-13). Accounting for radial anisotropy would thus strengthen our results (i.e., force $\beta$ downward). Tangential bias at these radii is quite rare; however, Sand et al. (2004) considered this possibility and estimated $\beta$ would be 
increased by $\lesssim 0.2$ in the clusters they studied. We conclude that our results are robust to any plausible level of orbital anisotropy.

In Section 4.2, possible systematic errors stemming from template mismatch and uncertain continuum fitting were found for our dispersion measurements at the $3 \%$ level. Additionally, we note a possible systematic difference of up to $\sim 10 \%$ for measurements on either side of the BCG center. Since our basic findings were unchanged when the dispersions were increased by 15\% (Section 5.1), we do not believe our results are substantially impacted by these uncertainties.

\subsection{Intracluster Gas}

We have neglected the intracluster gas in our mass models, and our measured dark matter profiles therefore include a small contribution from it. Allen et al. (2008) concluded from X-ray studies that the average gas mass fraction within $r_{2500} \approx 500 \mathrm{kpc}$ was $0.10 \pm 0.01$ in Abell 611, which decreases toward smaller radii. To assess the impact of neglecting gas on $\lesssim 20 \mathrm{kpc}$ scales, we subtracted the gas profile (kindly provided by S. Allen) from our gNFW models and found the slope was unchanged $(\Delta \beta \sim 0.01)$, as expected based on the very low gas fraction at small radii (Figure 11). We caution that this is based on an inward extrapolation of the gas profile, which is not measured for $r<17 \mathrm{kpc}$.

\subsection{Other Observational and Modeling Errors}

We have verified that our results on $\beta$ are not overly sensitive to the measured BCG size $\left(R_{e}\right.$ and $\left.r_{\text {cut }}\right)$ by perturbing it by the systematic error estimated in Table 3 . We have also made gross changes to seeing by perturbing it by $30 \%$. In all cases, the upper $68 \%$ confidence limit on $\beta$ increased $<0.04$. Finally, we note that although introducing ellipticity via the potential can produce unphysical negative surface densities at large radii (i.e., the outer regions of our weak lensing data) when $r_{s}$ is sufficiently small (Golse \& Kneib 2002; Sand et al. 2008), we checked that the reduced shear nevertheless remains physical in our models.

\section{DISCUSSION}

Our analysis of the mass density profile of Abell 611 over an unprecedented range in radii gives two fundamental results: an NFW profile is inconsistent with the data, and the logarithmic slope of the inner dark matter density profile is $<0.3(68 \%)$. It should be noted that our methodology allows us to disentangle the relative contributions of dark matter and baryons to the mass density profile, and our measurements therefore refer truly to the dark matter component. However, the cosmological $N$-body simulations to which we compare our measurements do not include baryons. Although their impact is thought to be smaller in galaxy clusters than in galaxies, baryons can be gravitationally dominant in cluster cores and may thus alter the dark matter distribution, particularly the inner slope.

High-resolution, cosmological $\mathrm{N}$-body + gas dynamical simulations of galaxy clusters (Gnedin et al. 2004) and galaxies (Gustafsson et al. 2006; Abadi et al. 2009, but see Romano-Díaz et al. 2008) have shown that as baryons condense in the center, they deepen the potential and steepen the dark matter distribution. In this scenario, the discrepancy we find with NFW-like dark matter profiles would be enhanced.

Gnedin et al. (2004) confirmed that this steepening occurs whether or not baryon cooling (as well as star formation, supernova feedback, and heating by the extragalactic UV background) was included, although the effect is larger when cooling is allowed. Cooling is certainly very important in cluster cores, but modeling the degree of cooling is currently limited by poor knowledge of a variety of baryonic processes, including viscosity (e.g., Dolag et al. 2005; Puchwein et al. 2005) and AGN feedback (e.g., Puchwein et al. 2008). Gnedin et al. (2004) acknowledge the common "overcooling problem" in their simulations, which should therefore provide an upper limit to the steepening. They found that a simple model of adiabatic contraction, modified from the original model of Blumenthal et al. (1986), reasonably describes the behavior of dark matter in their simulations. This has been implemented in the public Contra code, which we used to estimate the response of the dark matter halo in Abell 611 to the BCG. We find the dark matter mass enclosed within $10 \mathrm{kpc}$, approximately the inner limit of the simulations, is enhanced by $\sim 45 \%$. Since the theoretical and modeling uncertainties are large, this clearly should be considered a rough figure. However, it does demonstrate that substantial contraction (steepening) of the dark matter is possible, and we note that this uncertainty may be comparable or larger than possible oppositely-directed biases from unmodeled triaxiality (Section 5.1).

Several researchers have suggested that other physical processes, particularly dynamical friction between infalling baryons and the host halo, may counter and surpass the effects of adiabatic contraction in galaxy clusters. This work has been either (semi)-analytic, or based on $N$-body simulations without hydrodynamics, cooling, or (typically) a cosmological context, but is nevertheless important to bear in mind. If dynamical friction acts as strongly as claimed, our results could then be explained with no contradiction to cosmological $N$-body simulations of dark matter: initial NFW halos would form flatter cores through their interaction with baryons. El-Zant et al. $(2001,2004)$ considered an initially clumpy distribution of baryons and found they were efficient at "heating" the halo via dynamical friction, which softened the cusp. Their treatment of pure baryon clumps as unstrippable point masses may, however, exaggerate this effect. Indeed, Ma \& Boylan-Kolchin (2004) found that infalling DM subhaloes, depending on their mass and concentration, could steepen or soften the net DM cusp, and Nipoti et al. (2004) found a similar result depending on the baryon fraction of infalling galaxies. The mechanism by which the baryon clumps maintain their energy over sufficient timescales, without fragmenting and forming stars, is also unclear (Mashchenko et al. 2006). As a way of understanding its "universality," Gao et al. (2004) suggested that the NFW profile is a dynamical attractor, to which collisionless matter (stars and $\mathrm{DM}$ ) is driven in a hierarchical formation picture. Since stars are centrally dominant, this would imply a shallow DM profile. However, Gnedin et al. (2004) did not confirm this hypothesis in simulations including baryons. A recent analysis of a broad range of cosmological simulations (O. Y. Gnedin 2009, private communication) - including a variety of conditions and detailed treatments of the relevant physics-showed that baryonic physics can increase the range of contraction, but does not change the sign, i.e. does not lead to "expansion."

We thus conclude that our observations are hard to reconcile with the current understanding of cluster formation in the context of a $\Lambda \mathrm{CDM}$ cosmology. Either some of the assumptions about the dark matter backbone are incorrect or-more likely in our opinion, given the number of independent lines of evidence supporting CDM on large scales - baryonic physics is not yet sufficiently well understood. Given the centrality of this problem 

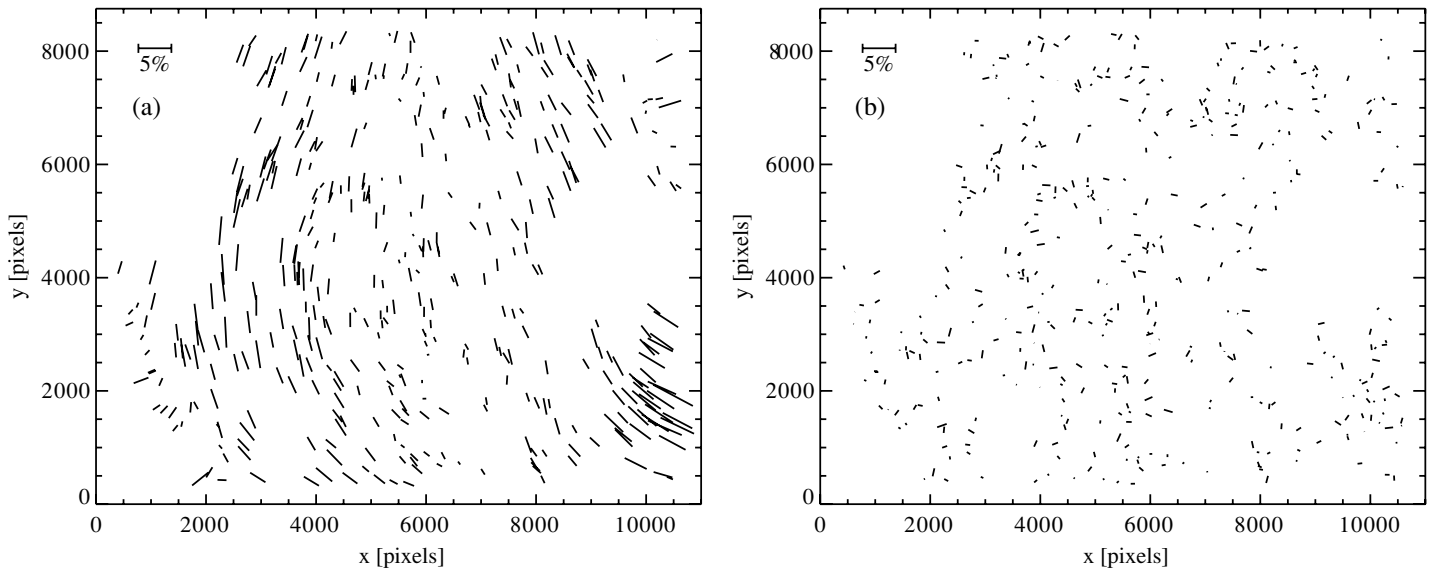

Figure 13. (a) Stellar ellipticities showing large-scale, smooth variation of the PSF across the focal plane. Light regions correspond to the excluded CCD chip (upper left) and masked stellar halos. (b) Residual ellipticities, small and pattern-free, after subtracting the polynomial model described in the text.

and the success of CDM at other scales, this seems a worthy goal to pursue, although its solution will likely require advances in computing power, algorithms, and understanding of the relevant physics.

A final caveat is that cluster mass density profiles may not be universal. Rather, a broader distribution of inner slopes may exist in nature than that predicted by pure dark matter simulations, potentially depending on the merger history of the cluster (see Navarro et al. 2008, and suggestions by our previous analysis of three clusters in Sand et al. 2004). If that is the case, it is imperative to collect data for a larger sample of clusters, of similar quality to those presented here for Abell 611, in order to characterize the moments of the distribution as well as the mean. Ideally the next generation of simulations including baryons should be powerful enough to enable the simulation of large numbers of clusters and therefore allow a comparison with the measured distribution of inner slopes, taking into account selection effects.

\section{SUMMARY}

We have constrained the DM profile of Abell 611 from $3 \mathrm{kpc}$ to $3 \mathrm{Mpc}$ and find that an NFW profile cannot simultaneously fit our lensing and kinematic data. We confirm the necessity of using mass probes over a wide range in cluster-centric radius to make the strongest comparisons with theory. Freeing the DM inner slope $\beta$ in our models increases the evidence by a factor of $2 \times 10^{4}$ (i.e., at more than $99 \%$ confidence) and selects a shallow slope $\beta<0.3$ (68\%). We intend to apply our technique to a wider sample of clusters in order to test the universality of our findings and measure the intrinsic scatter in cluster mass distributions.

We are grateful to Steve Allen for kindly providing X-ray measurements of the Abell 611 gas profile, and to Hervé Aussel, Hisanori Furusawa, and Yutaka Komiyama for assistance with photometric calibration. We thank Eric Jullo, Jason Rhodes, Joel Berge, Jean-Paul Kneib, Graham Smith, Richard Massey, and Simon White for their assistance and stimulating discussion. We acknowledge the anonymous referee for helpful suggestions. R.S.E. acknowledges financial support from the Royal Society. J.R. acknowledges support from an EU Marie Curie fellowship. T.T. acknowledges support from the NSF through CAREER award NSF-0642621, by the Sloan Foundation through a Sloan Research Fellowship, and by the Packard Foundation through a Packard Fellowship. The authors wish to recognize and acknowledge the cultural role and reverence that the summit of Mauna Kea has always had within the indigenous Hawaiian community. We are most fortunate to have the opportunity to conduct observations from this mountain. This research has made use of the NASA/IPAC Extragalactic Database, which is operated by the Jet Propulsion Laboratory, California Institute of Technology, under contract with the National Aeronautics and Space Administration.

\section{APPENDIX A}

\section{POINT-SPREAD FUNCTION}

In order to measure the small shape distortions to background galaxies in the weak lensing regime, we must correct the effects of the point-spread function (PSF), which is anisotropic and varies across the focal plane. We select bright but unsaturated stars from the stellar branch of the $R_{C}-r_{h}$ plane, where $r_{h}$ is the half-light radius, and use getshapes from the IMCAT suite to measure ellipticities and polarization tensors. Our method is based on that of Kaiser et al. (1995, KSB). In this scheme, shape measurements are performed with a window function to avoid formally infinite noise properties. We choose a Gaussian window with $\sigma=r_{g}=r_{h} /(2 \ln 2)^{1 / 2}$, which is optimal in the case of a Gaussian source. For stellar measurements, $r_{g}$ is fixed at the stellar median of $\left\langle r_{o}^{*}\right\rangle=1.8$ pixels $(0 ! 36)$. For galaxies, this is the minimum allowed $r_{g}$. For every object, the local (constant) background as estimated by SEXTRACTOR is subtracted. Ellipticities are then defined by $e_{\alpha}=\left\{Q_{11}-\right.$ $\left.Q_{22}, Q_{12}\right\} /\left(Q_{11}+Q_{22}\right)$, where $Q_{\alpha \beta}$ are the weighted quadrupole moments.

Stars used for PSF measurement must have (1) $\operatorname{tr} P_{s m}^{*}>0$ and $\operatorname{tr} P_{s h}^{*}>0$, where $P_{s m}^{*}$ and $P_{s h}^{*}$ are the smear and shear polarizability tensors, respectively, (2) no masked pixels or pixels assigned by SExTRACTOR to neighbors within $2 r_{g}$, (3) $|e|<0.2$, and (4) $d<0.1$ pixel, where $d$ is the distance between the centroid computed with and without a weight function, as discussed in Section 2.3.

A two-dimensional polynomial of degree 7 is fit to $e_{1}^{*}, e_{2}^{*}$, and $\operatorname{tr} P_{s m}^{*}$ with iterative $\sigma$-clip rejection and used to interpolate the 422 stars throughout the field. The measured stellar ellipticity field and the polynomial fit residuals are shown in Figure 13. Note that the large-scale, smooth PSF variations are removed. The PSF variance is reduced from $\sigma_{e_{\alpha}} \approx 0.01$ to $\approx 5 \times 10^{-3}$. At the position of each object, the interpolated stellar anisotropy 
kernel $q_{\alpha}^{*}$ is computed using the trace approximation to $P_{s m}^{*}$ :

$$
q_{\alpha}^{*}=e_{\alpha}^{*} /\left(\frac{1}{2} \operatorname{tr} P_{s m}^{*}\right) .
$$

The anisotropy-corrected ellipticity $e^{\prime}$ is then

$$
e_{\alpha}^{\prime}=e_{\alpha}-P_{s m, \alpha \alpha} q_{\alpha}
$$

where $P_{s m}$ is assumed diagonal.

\section{APPENDIX B}

\section{GALAXY SHAPE MEASUREMENT}

In addition to the anisotropy introduced by the PSF and corrected with Equation A2, galaxy ellipticities must be corrected for the isotropic smearing caused by seeing and the window function. This is done using the preseeing shear polarizability $P^{\gamma}$ described by Luppino \& Kaiser (1997). We use the trace approximation

$$
P^{\gamma}=\frac{1}{2}\left(\operatorname{tr} P_{\mathrm{sh}, \alpha \beta}-\operatorname{tr} P_{\mathrm{sm}, \alpha \beta}\left\langle\frac{\operatorname{tr} P_{\mathrm{sh}, \alpha \beta}^{*}}{\operatorname{tr} P_{\mathrm{sm}, \alpha \beta}^{*}}\right\rangle\right),
$$

where the quantity in angled brackets is the median stellar value. Since $P^{\gamma}$ is a noisy estimator, we correct galaxy shapes using an average value over galaxies with similar properties. Specifically, $P^{\gamma}$ is fit to a three-dimensional polynomial that is quadratic in $R_{C}, r_{h}$, and $|e|$, with iterative rejection. We find this is adequate to model the variation in $P^{\gamma}$. The interpolated $P^{\gamma}$ is used to estimate the reduced shear $g_{\alpha}=\gamma_{\alpha} /(1-\kappa)$ by

$$
g_{\alpha}=e_{\alpha}^{\prime} / P^{\gamma} \text {. }
$$

The uncertainty in $g_{\alpha}$ is also estimated from like galaxies. For each galaxy, the nearest 50 neighbors in the $R_{C}-r_{h}$ plane are selected, where the distance along each axis is normalized by the standard deviation. The dispersion in $g_{\alpha}$ among these neighbors is taken as the uncertainty. We note that this uncertainty (median $\left.\sigma_{g}=0.24\right)$ is dominated by the randomly distributed intrinsic shapes of galaxies.

\section{REFERENCES}

Abadi, M. G., Navarro, J. F., Fardal, M., Babul, A., \& Steinmetz, M. 2009, arXiv:0902.2477

Adelman-McCarthy, J. K., et al. 2008, ApJS, 175, 297

Alam, S. M. K., Bullock, J. S., \& Weinberg, D. H. 2002, ApJ, 572, 34

Allen, S. W., Rapetti, D. A., Schmidt, R. W., Ebeling, H., Morris, R. G., \& Fabian, A. C. 2008, MNRAS, 383, 879

Bartelmann, M., \& Meneghetti, M. 2004, A\&A, 418, 413

Barth, A. J., Ho, L. C., \& Sargent, W. L. W. 2002, AJ, 124, 2607

Benítez, N. 2000, ApJ, 536, 571

Bernardi, M., et al. 2003, AJ, 125, 1849

Bertin, E., \& Arnouts, S. 1996, A\&AS, 117, 393

Binney, J. J., \& Evans, N. W. 2001, MNRAS, 327, L27

Binney, J., \& Tremaine, S. 1987, Galactic Dynamics (1st ed.; Princeton, NJ: Princeton Univ. Press)

Biviano, A., \& Salucci, P. 2006, A\&A, 452, 75

Blumenthal, G. R., Faber, S. M., Flores, R., \& Primack, J. R. 1986, ApJ, 301, 27

Bradač, M., et al. 2005, A\&A, 437, 49

Bradač, M., et al. 2008, ApJ, 681, 187

Capak, P., et al. 2007, ApJS, 172, 99

Clowe, D., Luppino, G. A., Kaiser, N., Henry, J. P., \& Gioia, I. M. 1998, ApJ, 497, L61

Côté, S., Carignan, C., \& Freeman, K. C. 2000, AJ, 120, 3027

Crawford, C. S., Edge, A. C., Fabian, A. C., Allen, S. W., Bohringer, H., Ebeling, H., McMahon, R. G., \& Voges, W. 1995, MNRAS, 274, 75
Debattista, V. P., Moore, B., Quinn, T., Kazantzidis, S., Maas, R., Mayer, L., Read, J., \& Stadel, J. 2008, ApJ, 681, 1076

Diaferio, A., Geller, M. J., \& Rines, K. J. 2005, ApJ, 628, L97

Diemand, J., Moore, B., \& Stadel, J. 2004, MNRAS, 353, 624

Diemand, J., Zemp, M., Moore, B., Stadel, J., \& Carollo, C. M. 2005, MNRAS, 364,665

Dolag, K., Vazza, F., Brunetti, G., \& Tormen, G. 2005, MNRAS, 364, 753

Donovan, D. 2007, PhD thesis, Univ. Hawai'i

Elíasdóttir, Á., et al. 2007, arXiv:0710.5636

El-Zant, A. A., Hoffman, Y., Primack, J., Combes, F., \& Shlosman, I. 2004, ApJ 607, L75

El-Zant, A., Shlosman, I., \& Hoffman, Y. 2001, ApJ, 560, 636

Erben, T., Van Waerbeke, L., Bertin, E., Mellier, Y., \& Schneider, P. 2001, A\&A, 366,717

Gao, L., Loeb, A., Peebles, P. J. E., White, S. D. M., \& Jenkins, A. 2004, ApJ, 614,17

Gavazzi, R. 2005, A\&A, 443, 793

Gentile, G., Burkert, A., Salucci, P., Klein, U., \& Walter, F. 2005, ApJ, 634, L145

Gerhard, O., Jeske, G., Saglia, R. P., \& Bender, R. 1998, MNRAS, 295, 197

Gerhard, O., Kronawitter, A., Saglia, R. P., \& Bender, R. 2001, AJ, 121, 1936

Ghigna, S., Moore, B., Governato, F., Lake, G., Quinn, T., \& Stadel, J. 2000, ApJ, 544,616

Gnedin, O. Y., Kravtsov, A. V., Klypin, A. A., \& Nagai, D. 2004, ApJ, 616, 16

Golse, G., \& Kneib, J.-P. 2002, A\&A, 390, 821

Gustafsson, M., Fairbairn, M., \& Sommer-Larsen, J. 2006, Phys. Rev. D, 74, 123522

Hauschildt, P. H., Baron, E., \& Allard, F. 1997, ApJ, 483, 390

Hoekstra, H. 2003, MNRAS, 339, 1155

Jing, Y. P., \& Suto, Y. 2002, ApJ, 574, 538

Jullo, E., Kneib, J.-P., Limousin, M., Elíasdóttir, Á., Marshall, P. J., \& Verdugo, T. 2007, New J. Phys., 9, 447

Kaiser, N., Squires, G., \& Broadhurst, T. 1995, ApJ, 449, 460

Kassiola, A., \& Kovner, I. 1993, ApJ, 417, 450

Kelson, D. D., Zabludoff, A. I., Williams, K. A., Trager, S. C., Mulchaey, J. S., \& Bolte, M. 2002, ApJ, 576, 720

Kneib, J. P., Mellier, Y., Fort, B., \& Mathez, G. 1993, A\&A, 273, 367

Kneib, J.-P., et al. 2003, ApJ, 598, 804

Krist, J. 1993, in ASP Conf. Ser. 52, Astronomical Data Analysis Software and Systems II, ed. R. J. Hanisch, R. J. V. Brissenden, \& J. Barnes (San Francisco, CA: ASP), 536

Kronawitter, A., Saglia, R. P., Gerhard, O., \& Bender, R. 2000, A\&AS, 144, 53

Kuzio de Naray, R., McGaugh, S. S., \& de Blok, W. J. G. 2008, ApJ, 676, 920

Landolt, A. U. 1992, AJ, 104, 340

Limousin, M., et al. 2008, A\&A, 489, 23

Luppino, G. A., \& Kaiser, N. 1997, ApJ, 475, 20

Ma, C.-P., \& Boylan-Kolchin, M. 2004, Phys. Rev. Lett., 93, 021301

Mahdavi, A., Hoekstra, H., Babul, A., Sievers, J., Myers, S. T., \& Henry, J. P. 2007, ApJ, 664, 162

Marchesini, D., D’Onghia, E., Chincarini, G., Firmani, C., Conconi, P., Molinari, E., \& Zacchei, A. 2002, ApJ, 575, 801

Marshall, P. J., Hobson, M. P., Gull, S. F., \& Bridle, S. L. 2002, MNRAS, 335 , 1037

Mashchenko, S., Couchman, H. M. P., \& Wadsley, J. 2006, Nature, 442, 539

Massey, R., et al. 2007, MNRAS, 376, 13

McGaugh, S. S., de Blok, W. J. G., Schombert, J. M., Kuzio de Naray, R., \& Kim, J. H. 2007, ApJ, 659, 149

Meneghetti, M., Bartelmann, M., Jenkins, A., \& Frenk, C. 2007, MNRAS, 381 , 171

Miralda-Escude, J. 1995, ApJ, 438, 514

Mo, H. J., \& Mao, S. 2000, MNRAS, 318, 163

Mobasher, B., et al. 2007, ApJS, 172, 117

Monet, D. G., et al. 2003, AJ, 125, 984

Moore, B., Governato, F., Quinn, T., Stadel, J., \& Lake, G. 1998, ApJ, 499, L5

Natarajan, P., Kneib, J.-P., Smail, I., Treu, T., Ellis, R., Moran, S., Limousin, M., \& Czoske, O. 2009, ApJ, 693, 970

Navarro, J. F., Frenk, C. S., \& White, S. D. M. 1996, ApJ, 462, 563

Navarro, J. F., Frenk, C. S., \& White, S. D. M. 1997, ApJ, 490, 493

Navarro, J. F., \& Steinmetz, M. 2000, ApJ, 528, 607

Navarro, J. F., et al. 2004, MNRAS, 349, 1039

Navarro, J. F., et al. 2008, MNRAS, submitted (arXiv:0810.1522)

Neto, A. F., et al. 2007, MNRAS, 381, 1450

Nipoti, C., Treu, T., Ciotti, L., \& Stiavelli, M. 2004, MNRAS, 355, 1119

Peng, C. Y., Ho, L. C., Impey, C. D., \& Rix, H.-W. 2002, AJ, 124, 266

Puchwein, E., Bartelmann, M., Dolag, K., \& Meneghetti, M. 2005, A\&A, 442, 405

Puchwein, E., Sijacki, D., \& Springel, V. 2008, ApJ, 687, L53 
Richard, J. 2009, ApJ, submitted

Romano-Díaz, E., Shlosman, I., Hoffman, Y., \& Heller, C. 2008, ApJ, 685, L105

Romanowsky, A. J., \& Kochanek, C. S. 1998, ApJ, 493, 641

Saglia, R. P., Kronawitter, A., Gerhard, O., \& Bender, R. 2000, AJ, 119, 153

Salucci, P. 2001, MNRAS, 320, L1

Sand, D. J., Treu, T., \& Ellis, R. S. 2002, ApJ, 574, L129

Sand, D. J., Treu, T., Ellis, R. S., Smith, G. P., \& Kneib, J.-P. 2008, ApJ, 674 711

Sand, D. J., Treu, T., Smith, G. P., \& Ellis, R. S. 2004, ApJ, 604, 88

Schlegel, D. J., Finkbeiner, D. P., \& Davis, M. 1998, ApJ, 500, 525

Schmidt, R. W., \& Allen, S. W. 2007, MNRAS, 379, 209

Simon, J. D., Bolatto, A. D., Leroy, A., \& Blitz, L. 2003, ApJ, 596, 957

Skrutskie, M. F., et al. 2006, AJ, 131, 1163

Smith, G. P., Kneib, J.-P., Smail, I., Mazzotta, P., Ebeling, H., \& Czoske, O. 2005, MNRAS, 359, 417

Springel, V., Frenk, C. S., \& White, S. D. M. 2006, Nature, 440, 1137
Swaters, R. A., Madore, B. F., van den Bosch, F. C., \& Balcells, M. 2003, ApJ, 583,732

Thomas, J., Saglia, R. P., Bender, R., Thomas, D., Gebhardt, K., Magorrian, J., Corsini, E. M., \& Wegner, G. 2005, MNRAS, 360, 1355

Thomas, J., Saglia, R. P., Bender, R., Thomas, D., Gebhardt, K., Magorrian, J., Corsini, E. M., \& Wegner, G. 2007, MNRAS, 382, 657

Treu, T., \& Koopmans, L. V. E. 2004, ApJ, 611, 739

Treu, T., Stiavelli, M., Casertano, S., Møller, P., \& Bertin, G. 1999, MNRAS, 308, 1037

Umetsu, K., \& Broadhurst, T. 2008, ApJ, 684, 177

Umetsu, K., et al. 2009, ApJ, 694, 1643

van der Marel, R. P. 1994, MNRAS, 270, 27

van Dokkum, P. G. 2001, PASP, 113, 1420

Young, P. J. 1976, AJ, 81, 807

Zappacosta, L., Buote, D. A., Gastaldello, F., Humphrey, P. J., Bullock, J., Brighenti, F., \& Mathews, W. 2006, ApJ, 650, 777 